\title{
Altered Calcium Permeability of AMPA Receptor Drives NMDA Receptor Inhibition in the Hippocampus of Murine Obesity Models
}

\section{Yasuyo Miyagi}

University of the Ryukyus: Ryukyu Daigaku

\section{Kyoko Fujiwara}

University of the Ryukyus: Ryukyu Daigaku

\section{Keigo Hikishima}

OIST: Oriental Institute of Science and Technology

\section{Daisuke Utsumi}

University of the Ryukyus: Ryukyu Daigaku

\section{Chiaki Katagiri}

University of the Ryukyus: Ryukyu Daigaku

\section{Masahiko Nlshimura}

University of the Ryukyus: Ryukyu Daigaku

Hiroshi Takagi

University of the Ryukyus: Ryukyu Daigaku

Shogo Ishiuchi ( $\nabla$ ishogo@med.u-ryukyu.ac.jp )

University of theRyukyus https://orcid.org/0000-0002-8967-2019

\section{Research Article}

Keywords: Obesity, CP-AMPAR, NMDAR, Cognitive function

Posted Date: December 29th, 2021

DOI: https://doi.org/10.21203/rs.3.rs-1200714/v1

License: (c) (1) This work is licensed under a Creative Commons Attribution 4.0 International License. Read Full License 


\section{Abstract}

Evidence has accumulated that higher consumption of high-fat diets (HFDs) during the juvenile/adolescent period induces altered hippocampal function and morphology; however, the mechanism behind this phenomenon remains elusive. Using high-resolution structural imaging combined with molecular and functional interrogation, a murine model of obesity treated with HFDs for 12 weeks after weaning mice was shown to change in the glutamate-mediated intracellular calcium signaling and activity, including further selective reduction of gray matter volume in the hippocampus associated with memory recall disturbance. Dysregulation of intracellular calcium concentrations was restored by a noncompetitive a-3-hydroxy-5-methyl-4-isoxazole propionic acid receptor (AMPAR) antagonist, followed by normalization of hippocampal volume and memory recall ability, indicating that AMPARs may serve as an attractive therapeutic target for obesity-associated cognitive decline.

\section{Introduction}

The global obesity epidemic has put forth evidence that high-fat diets (HFDs) (40-65\% kcal derived from fat) or simple sugars (sucrose or high fructose corn syrup) promote obesity, which has a negative effect on neurocognition [1,2]. Early life exposure to obesogenic diets like simple sugars and saturated fatty acids induce learning and memory disturbance, which is presumed to be based on increased neuroinflammation and reduced neurotrophin mediated regulation of neurogenesis and synaptic plasticity [1]. Furthermore, a western diet with dense energy including saturated fat and simple sugar intake could invoke a vicious cycle of hippocampal dysfunction and impaired feeding behavior, stimulates excess intake, obesity, and resulting in hippocampal dysfunction, which further accelerates obesity, followed by cognitive dysfunction [2]. To our great concern, HFDs during the juvenile/adolescent period induce alterations in hippocampal morphology and function [3-6]. Several evidence accumulated various hippocampal dysfunction such as synaptic plasticity of long-term potentiation (LTP) and longterm depression (LTD) with obese HFD-treated adult male-but not female-mice [3], and spatial learning in the radial-arm maze [4] by HFDs. The impairment of LTD was identified as the most relevant change in synaptic plasticity due to HFD consumed during the juvenile period [4]. These apparent alterations of hippocampus synaptic plasticity associated with spatial and memory disturbance strongly suggest an influence of HFDs on glutamatergic neurotransmission [6].

AMPARs as well as NMDARs play a crucial role for these forms of synaptic plasticity $[7,8]$. We identified $\mathrm{Ca}^{2+}$-permeable AMPAR (CP-AMPAR) is related to the pathophysiology in neurological disorders such as brain tumor [9], and also relevant to amyotrophic lateral sclerosis (ALS) [10], cocaine addiction [11], neuropathic pain [12], and epilepsy [13]. Changes in the expression of CP- AMPARs can alter synaptic properties, lead to damage of selectively vulnerable neurons $[7,8]$ CP-AMPARs are more widespread than originally thought and play a role in synaptic plasticity and neuronal death, though $\mathrm{Ca}^{2+}$ influx through AMPARs is modest compared with that through NMDARs[8, 14]. An issue of functional consequences of AMPA receptor mediated $\mathrm{Ca}^{2+}$ signaling remains elusive in post synaptic membrane. Although 
accumulated evidence indicated that AMPA receptor mediated $\mathrm{Ca}^{2+}$-influx modulates efficacy of fast synaptic transmission and can trigger LTP, relatively little is known about the postsynaptic target of $\mathrm{Ca}^{2+}$ [8]. Fast interaction between AMPA and NMDA receptors by intracellular calcium was experimentally observed by introduction of recombinant CP-AMPAR and $\mathrm{Ca}^{2+}$-impermeable NMDAR mutant channels into HEK 293 [15]. $\mathrm{Ca}^{2+}$-entry through AMPARs inactivates co-localized NMDARs in the time range of excitatory postsynaptic currents, but the significance of this phenomenon in neurological disorders is unknown. In this study, we show here role of AMPAR-mediated $\mathrm{Ca}^{2+}$-entry in obesity mice. NMDAR blocked by this CP-APMAR induces spatial and social memory disturbance in these mice.

Hippocampal atrophy was affected by obesity, and type II diabetes mellitus as well as Alzheimer disease, traumatic brain injury, obstructive sleep apnea, and depression [5]. Using high-resolution structural imaging combined with molecular and functional interrogation, in a male murine model of obesity treated with HFDs for 12 weeks after weaning, we examined changes in the glutamate-mediated intracellular calcium signaling and activity, volumetric analysis of hippocampal subregions as well as the rest of brain, and behavioral estimation. Both murine models of obesity showed similar changes in the glutamate-mediated intracellular calcium signaling and activity associated with memory recall disturbance. In the quantitative volumetric analysis of the brain by $11.7 \mathrm{~T}$ magnetic resonance imaging (MRI) (Bruker BioSpec 117/11, Bruker BioSpin GmbH, Germany), we found a notable sign of brain atrophy caused by the reduction of brain volume. We discovered for the first time that altered calcium permeability of AMPA receptor drives NMDA receptor inhibition in the acute slice of hippocampus in these disease models. Furthermore, dysregulation of intracellular calcium concentrations was restored by a non-competitive AMPAR antagonist [16], followed by normalization of hippocampal volume and memory recall ability, indicating that AMPARs may serve as an attractive therapeutic target for obesity-associated cognitive decline.

Lastly, we examined whether similar findings in murine obese models were adopted in humans, we conducted volumetric analysis using MRI (Discovery MR750; General Electric, Milwaukee, WI) T2 data for overweight and obese people compared with that of normal-weight volunteers. BMI impacts negatively on human gray matter volume, interaction with default mode network(DMN) and anti- DMN dynamics, and network connectivity of hippocampal memory circuits $[17,18]$.

\section{Materials And Methods}

\section{Animals}

Male C57BL/6J background mice were obtained from CLEA Japan Inc. and Japan SLC, respectively, because male mice are reported to be more vulnerable to the effects of HFD on weight gain, metabolic alterations and deficits of learning, and hippocampal synaptic plasticity. These animals were housed three or four per cage in a standard $12 \mathrm{~h}$ dark-light cycle room at $25^{\circ} \mathrm{C}$ with freely available food and water. All animal experiments were performed in accordance with the guidelines of the Animal Experiment 
Ethics Committee of the University of the Ryukyus (approval number: A2019239). Time series changes in average food intake and average body weight in each condition were monitored.

\section{Feed for control and high fat diet}

In the case of the control diet (CD) for mice, we used CE-2 feed (Japan CLEA, Tokyo, Japan). The composition table of CE-2 feed is shown in Table S1; the total energy was $339.1 \mathrm{kcal} / 100 \mathrm{~g}$, and crude fat was $4.61 \%$. In contrast, in the case of high-fat diets (HFDs), we used F2HFD2 feed (Oriental Yeast Co., Ltd., Tokyo, Japan). The total energy of F2HFD2 was $640 \mathrm{kcal}$, consisting of 58\% lard (wt/wt), 30\% fish powder, $10 \%$ skim milk, and a $2 \%$ vitamin and mineral mixture (equivalent to $7.5 \%$ carbohydrate, $24.5 \%$ protein, and 60\% fat)[19]. The other components of F2HFD were similar to those of CE-2. At 4 weeks of age, groups of mice were subjected to HFDs (F2HFD2 feed). Control mice were fed a low-fat diet (CE-2 feed).

\section{Oral delivery of perampanel (PER)}

HFD mice were orally administered with HydroGel (Clear $\mathrm{H}_{2} \mathrm{O}$, Portland, ME 04101, USA) at a dose of 5 $\mathrm{mg} / \mathrm{kg}$ body weight (HFD-fed mice with PER treatment group). In every HFD mice, one in one cage, PER was orally administered at a dose of $5 \mathrm{mg} / \mathrm{kg}$ body weight (HFD mice with PER treatment group), and both groups were monitored for body weight and intake of HydroGel twice a week to adjust the administration dose of PER to $5 \mathrm{mg} / \mathrm{kg}$ per body weight.

\section{Wheel-running activity}

Wheel cages (MELQUEST, Japan, Model RWC-15) were used to monitor individual mouse activity. Wheel rotation was monitored and recorded every $10 \mathrm{~min}$ for 14 days, as reported previously [20].

\section{Open field test and elevated plus maze}

Open field test: In a space surrounded by a $50 \mathrm{~cm}$ square and $40 \mathrm{~cm}$ high wall (Muromachi Kikai, Japan), mice were allowed to act freely for $5 \mathrm{~min}$, and the trajectory was analyzed using a CompACT VAS/DV video-tracking system (Muromachi Kikai, Japan) [21]. Elevated plus maze: A space was elevated to a height of $50 \mathrm{~cm}$ from the floor, consisting of two open arms, two closed arms (30 × $6 \mathrm{~cm}$ each), and a neutral zone. Mice were placed in the center of the neutral zone, facing a closed arm, and allowed to move freely for $3 \mathrm{~min}$. The time spent in the open and closed arms and the frequency of visits to the different arms were recorded and scored using the CompACT VAS/DV video-tracking system (Muromachi Kikai, Japan).

\section{Novel object recognition test}

Mice with CD, HFDs, and HFDs with PER treatment were handled for 5 min daily for 5 days prior to the start of the novel object recognition test. The mice were habituated to a $35 \mathrm{~cm}$ square and $25 \mathrm{~cm}$ high box for 10 min on day 1 and were provided with two identical objects (familiar object) for 10 min on day 2. On day 3 , one of the familiar objects was exchanged with a novel object of different shape and color 
(Fig. 2f). The behavior of the mice was monitored for 5 min using a video camera, and the videos were digitized and stored on a personal computer. The search time for each object was measured via offline analysis [22].

\section{Morris water maze}

Memory impairment was assessed using the Morris water maze test, as previously described [23]. In summary, the water maze pool (Muromachi Kikai, Tokyo, Japan), with a diameter of $120 \mathrm{~cm}$, contained opaque water (room temperature) with a platform (10 cm in diameter) submerged $2 \mathrm{~cm}$ below the surface. The hidden platform task took 4-7 days (two sessions per day, 3 hours apart), during which two trials were performed each day (15 min apart). The platform location remained constant, and the entry points were changed semi-randomly between the trials. Twenty-four hours after the last day of the hidden platform task, a 1 min probe trial was performed without the platform. The entry point for the probe trials was in the quadrant opposite to the target quadrant. Memory retention was evaluated by the amount of time spent in the correct quadrant where the escape platform was located in the hidden platform trial. The performance was monitored using the CompACT VAS/DV video-tracking system.

\section{Pattern completion test mediated contextual and spatial recall}

The Morris water maze task was conducted in mice with CD, HFDs, and HFDs with PER treatment as described previously [24]. All experiments were conducted at approximately the same time of day. The mice were transported from the colony to a holding area, where they were undisturbed for 30 min prior to the experiment. The test was performed in a rectangular dimly lit room with a circular pool (Muromachi Kikai, Tokyo, Japan), with a diameter of $120 \mathrm{~cm}$, filled with opaque water made with skim milk (Morinaga, Japan) maintained at room temperature. Four large objects illuminated with floor lamps were hung on black curtains surrounding the pool as extramaze cues. A hidden circular platform ( $10 \mathrm{~cm}$ in diameter) was placed $1 \mathrm{~cm}$ below the water surface, and the mice were trained to find the platform with four trials per day for 12 days, with an inter-trial interval of approximately $60 \mathrm{~min}$. During training, the mice were released from four pseudorandomly assigned start locations ( $N, S, E$, and $W$ ) and were allowed to swim for $300 \mathrm{~s}$. If a mouse did not find the platform within $300 \mathrm{~s}$, it was manually guided to the platform and allowed to rest on the platform for $15 \mathrm{~s}$.

A probe trial was conducted on day 13 under the full-cue condition (P1). The mice were released at the center of the pool and allowed to swim for $300 \mathrm{~s}$ in the absence of the platform. Following the probe trial, the mice received four training trials in the presence of the platform to avoid memory extinction that may have occurred during the probe trial. Subsequently, the mice received four probe trials with extra maze cue manipulations, one probe trial per day, without retraining between probe trials. For the one-cue probe trial (P2), one cue located more distally from the platform was kept, and the other three cues were removed from the surrounding curtains. For the two-cue probe trial (P3), one cue located close to the platform and the cue used in the one-cue probe trial remained, but the other two cues were removed from the surrounding curtains. For the no-cue probe trial (P5), all four extra maze cues were removed. Data for 
training and probe trials were collected and analyzed using the CompACT VAS/DV video-tracking system software. The escape latency to the hidden platform (goal arrival time) was measured.

\section{Five-trial social memory assay}

The five-trial social test was performed as described previously [25]. In summary, subject mice with CD, HFDs, and HFDs with PER treatment were individually housed for 7 days before testing to establish territorial dominance. On the day of testing, a female mouse was presented to the subject male mouse's cage (Fig. 2g) for four successive 5 min trials with a 10 min inter-trial interval. In the fifth trial, a novel female mouse was presented (Fig. 2g), and the duration of the social investigation was recorded.

\section{Contextual fear conditioning}

Contextual fear conditioning was applied according to published protocols with slight modifications [26]. The mice were transported to an animal experimental room and allowed to acclimatize for at least $30 \mathrm{~min}$ prior to contextual fear conditioning training. The mice were then placed in the foot shock system model MK-450MSQ (Muromachi Kikai Co. Ltd., Japan) and allowed to explore for 2 min followed by three electric foot shocks ( $0.8 \mathrm{~mA}, 2 \mathrm{~s}$, and 2 min intervals). Animals were left in the apparatus for a further minute before removal.

\section{Acquisition of MRI data for mice}

Anatomical brain images of 8 ex vivo mice with CD, HFDs, and HFDs with PER treatment were obtained using a Bruker BioSpec 117/11 11.75 Tesla MRI scanner (Bruker BioSpin GmbH, Ettlingen, Germany). A three-dimensional (3D)-prepared rapid gradient-echo (MPRAGE) sequence was acquired as highresolution $100 \mu \mathrm{m}$ isovoxel image of voxel-based morphometry (matrix size: $280 \times 220 \times 220$; field of view: $28 \times 22 \times 22 \mathrm{~mm}$; repetition time: $2000 \mathrm{~ms}$; echo time: $1.78 \mathrm{~ms}$; flip angle: 12 degrees; inversion time: $800 \mathrm{~ms}$; echo train length: 13; numbers of average: 2). A rapid acquisition protocol by relaxation enhancement (RARE) sequence was used to draw the region of interest in the acquired data image (matrix size: $280 \times 220 \times 220$; field of view: $28 \times 22 \times 22 \mathrm{~mm}$; repetition time: $1500 \mathrm{~ms}$; echo time: $25 \mathrm{~ms}$; flip angle: $180^{\circ}$ ). We obtained MPRAGE and RARE images simultaneously in 1 single scan. MRI data acquisition of ex vivo mice was obtained from the heads of four mice that were fixed with PBS into the MRI coil simultaneously. Brains of the four mice were confirmed using raw MRI data. Rodent MRI was performed at the University of the Ryukyus under the OIST Sign Cooperation Agreement (Research theme: Medical impacts of brain volume control effect for reserve capacity of brain atrophy and delay the onset of symptoms of cognitive impairment - By using high resolution MRI analysis system-).

\section{Image preprocessing and estimations for voxel-based morphometry analysis}

Voxel-based morphometry (VBM) analysis and preprocessing of MPRAGE and RARE images were performed using the SPM8 analysis tool (Wellcome Department of Clinical Neurology, London; http://www.fil.ion.ucl.ac.uk) and SPMMouse toolbox (http://www.spmmouse.org/). The raw data were divided into four mice head MRI images and stored separately. The 3D $\left(x-y-z^{-}\right)$coordinates of the 
divided images were transformed to the SPM standard coordinate system, and we defined the origin of the 3D coordinates as the bregma point. Then, the brain images were segmented into gray matter (GM), white matter, and cerebrospinal fluid using a segmentation tool (installed in the SPM8 system). The image was divided into GM images using tissue probability maps in the SPMMouse toolbox. These divided GM images were improved by contrast and normalized to the deformation of images. Finally, these images were smoothed using a $200 \mu \mathrm{m}$ isotropic Gaussian kernel method (installed in the SPM8 system). A smoothed image was applied to the VBM analysis. The volume of hippocampal subfields (CA1, CA2, CA3, DG, and EC) was calculated using the ROI files [18, 27-29]. The differences among the mean values of the whole brain volumes from CD, HFDs, and HFDs with PER treatment were tested by one-way analysis of variance (ANOVA). When there was a significant difference between the three groups by ANOVA ( $p<0.05)$, a Scheffe post hoc analysis (Scheffe) was performed between the CD vs. HFDs groups; CD vs. HFDs with PER treatment groups; and HFDs vs. HFDs with PER treatment groups.

\section{Human subjects}

The participants of this study were 117 healthy volunteers (mean age $37.8 \pm 19.6$ years; 65 females, 52 males) and five patients with benign tumors (mean age $55.5 \pm 9.6$ years; two females, three males), and all participants agreed in writing to participate in this study, in event-related memory tasks and T1weighted imaging by 3-TMRI. The participants were divided into three groups based on body mass index (BMI) according to the WHO criteria: normal weight (BMI < 25), overweight (BMI $\geq 25$ and $<30$ ), and obese (BMI $\geq 30$ ). There were 84 patients in the normal weight group (mean BMI $20 \pm 1.8$ ), 27 in the overweight group (mean BMI $26 \pm 1.2$ ), and 11 in the obese group (mean BMI $32 \pm 2.3$ ). All experiments were approved by the ethical committee of the University of the Ryukyus for medical and health research involving human subjects and were performed in accordance with guidelines of human experiment regulations at University of the Ryukyus (approval number:111).

\section{Behavioral task paradigm}

Details of the fMRI experiment of the event-related memory task used in this study are described in a previous report [17]. The memory task consisted of 108 photographs of 16 lure sets, 16 repeat sets, and 44 novel items. Participants were instructed to respond with buttons whether the photo stimulus shown on the display was a novel item (new), repeated photograph (same), or similar to but not the same as the previous photograph (lure). The button responses of the participants during the memory task were recorded on a personal computer, and the correct answer rates for new, same, and lure were calculated. The correct answer rate for each task (new task, lure task, and same task) was calculated using the following formula: The correct answer rate $(\%)=$ number of correct answers to the presented task $/$ total number of presented tasks $\times 100$. In our experiments, the total number of presented tasks for the new stimulus was set to 76 , and the total number of tasks for the same and lure stimuli were both set to 16 .

\section{Functional MRI data acquisitions for behavioral task}

Functional and structural images of the brain were obtained using 3T-MRI (Discovery MR750; General Electric, Milwaukee, WI). A sequence of echo planar imaging (EPI, repetition time: 1500 ms, echo time: 25 
ms, flip angle: $70^{\circ}$, matrix size: $128 \times 128$, field of view: $192 \times 192$, in-plane resolution: $1.5 \times 1.5 \mathrm{~mm}^{2}, 23$ slices, $3 \mathrm{~mm}$ thickness, $0 \mathrm{~mm}$ space) was used for the functional images for measuring BOLD contrast. The anatomical brain image was obtained using a three-dimensional (3D) spoiled gradient recalled echo (SPGR) sequence ( $1 \mathrm{~mm}$ slice thickness in sagittal section, matrix size: $256 \times 256$, field of view: $256 \times$ $256 \mathrm{~mm}$, repetition time: $6.9 \mathrm{~ms}$, echo time: $3 \mathrm{~ms}$, flip angle: $15^{\circ}$ ). A high-resolution T2-weighted fast spin echo sequence (matrix size: $512 \times 512$, field of view: $192 \times 192 \mathrm{~mm}$, repetition time: $4300 \mathrm{~ms}$, echo time: $92 \mathrm{~ms}$, in-plane resolution: $0.375 \times 0.375 \mathrm{~mm}^{2}, 23$ slices, $3 \mathrm{~mm}$ thickness, $0 \mathrm{~mm}$ space) was obtained for visualizing the hippocampal structure and co-registration of 3D SPGR images and EPI functional images.

\section{Imaging processing for behavioral task fMRI}

Realignment, temporal correlation, spatial normalization, and spatial smoothing of the functional images were preprocessed and analyzed using SPM12. After preprocessing, the BOLD contrast images of new, same, and lure conditions of the individual subject were calculated from the functional image data: the hippocampal subregions as the CA3, CA1 and DG, and the perihippocampus region as the parahippocampus gyrus, perirhinal cortex, and entorhinal cortex were drawn manually with a pen tablet on the high-resolution coronal T2-weighted image based on the hippocampus atlas [30]. The percentages of signal changes in the BOLD response in the hippocampal regions of each subject were extracted using the MarsBar toolbox.3D-SPGR images were used for the analysis of voxel-based morphometry. The T1weighted images were segmented into GM, white matter, and cerebrospinal fluid images using the SPM 12 segmentation tool. The volume of GM in the whole brain was calculated after spatial normalization and modulation of the GM image.

\section{Image acquisition for functional connectivity analysis}

MRI data were acquired using a GE Medical Discovery MR 750 3T scanner with a 32-channel head coil. Participants who lay on the scanner bed in the supine position were fixed to the head and neck by form pads and a Philadelphia neck collar to minimize head movement. Resting-state fMRI images were acquired using a single-shot EPI sequence covering the whole brain (42 axial slices, $4 \mathrm{~mm}$ thickness with no inter-slice gap; repetition time, $2000 \mathrm{~ms}$; echo time, $30 \mathrm{~ms}$; flip angle, $70^{\circ}$; matrix size, $64 \times 64$; field of view, $256 \times 256$ ). A total of 150 volumes were imaged over a single session. The anatomical brain images were acquired using T1-weighted, sagittal 3D SPGR sequences.

\section{Data preprocessing and analysis for functional network}

Image preprocessing and functional network analysis were performed using SPM12 and CONN toolbox 18.b (www.nitrc.org/projects/conn, RRID: SCR_009550) [31]. The details were described in a previous report [17]. Images were preprocessed in order of realignment, slice-timing correction, coregistration, normalization, smoothing, and segmentation. A noise of BOLD signal was removed by linear regression of potential confounding effects in the BOLD signal, and temporal band-pass filtering (temporal frequencies below $0.008 \mathrm{~Hz}$ or above $0.09 \mathrm{~Hz}$ ). 
We analyzed the default mode network (DMN) and functional connectivity in functional network analysis using the CONN toolbox. First as an individual analysis, regions with a positive correlation to BOLD fluctuations of a posterior cingulate cortex (PCC) and a precuneus were calculated as a DMN map. Next, regions with a negative correlation to the DMN map were calculated as anticorrelation DMN maps. Mean images of DMN map and anticorrelation DMN map of the normal weight, overweight, and obese groups were calculated using a one-sample t-test (voxel-level threshold of $p<0.001$ uncorrected, and a clusterlevel threshold of false discovery rate $[F D R]$ corrected $p<0.05)$. In the functional combination analysis, we computed the nodes and edges in the graph theory parameters. The correlation coefficient of the fluctuation of BOLD signal between each ROI was calculated for seeds using the $132 \times 132 \mathrm{ROI}$ map (default ROI atlas of CONN toolbox)-based seed-based connectivity measure [32]. The ROI-to-ROI degree (number of edges that any node has between each node) and betweenness centrality (the number of shortest paths of a vertex that passes to any two pairs $(j, i)$ of nodes within a graph) were calculated by an associated ROI-to-ROI correlation $132 \times 132$ matrix, established using our $132 \times 132 \mathrm{ROI}$ map. The degree is defined at each node as the number of edges from/to each node. Degree $\left(d_{i}\right)$ is defined as the following formula: $d i=\sum_{j} A i, j$. where $\mathrm{A}_{\mathrm{i}, \mathrm{j}}$ is the associated ROI-to-ROI correlation of the $132 \times 132$ matrix. $i=1,2,3 \ldots . ., 132 ; j=1,2,3 \ldots, 132$. Betweenness centrality $\left(B C_{i}\right)$ represents a hub that connects other

functional modules and nodes [31], and $B C_{i}$ is defined as follows: $B C_{i}=\frac{\sum_{j, k \neq i}\left[i \in P_{j, k}\right]}{(N-1)(N-2)}$. where $P_{j, k}$ is the number of nodes in the shortest path (shortest path when passing through any node defined with $i$ as a variable) between each pair $(k, j)$ of the nodes, and $N$ is the total number of nodes in a graph of our ROI map. $j, k \neq i ; N=i \times j=132^{2}$. We calculated the mean value of each node and the number of edges in the normal-weight, overweight, and obese groups, respectively. In addition to the PCC and the precuneus in the DMN, the anterior cingulate cortex, cerebellar lobules Crusl, and hippocampus related to hippocampal memory function were set as regions of interest as reported previously [17], and the functional connectivity of the three groups was analyzed. A functional connectivity map of each group was illustrated using the BrainNet viewer (https://www.nitrc.org/projects/bnv/) [33].

\section{Brain slices Ca imaging}

Hippocampal brain slices were prepared from 17-18-week-old male C57BL/6J background mice, which divided the mice into CD, HFDs, and HFDs with PER treatment groups according to previous reports [34]. After cutting the slices (thickness, 300-400 $\mu \mathrm{m}$ ) using the Linear Slicer (PRO 7N, Dosaka EM, Japan), hippocampal brain slices were incubated in the normal Krebs Ringer $(125 \mathrm{mM} \mathrm{NaCl}, 2.5 \mathrm{mM} \mathrm{KCl}, 10 \mathrm{mM}$ D-glucose, $1.25 \mathrm{mM} \mathrm{NaH}_{2} \mathrm{PO}_{4}, 26 \mathrm{mM} \mathrm{NaHCO}_{3}, 2 \mathrm{mM} \mathrm{CaCl}_{2}, 1 \mathrm{mM} \mathrm{MgCl}_{2}$, continuously bubbled by mixture gas $\left.\left[95 \% \mathrm{O}_{2} ; 5 \% \mathrm{CO}_{2}\right]\right)$ for $60 \mathrm{~min}$ at room temperature to recover the cutting damage. The $\mathrm{Ca}$ indicator Fluo-3 AM (excitation wavelength: $508 \mathrm{~nm}$, emission: $525 \mathrm{~nm}, \mathrm{Kd}, 0.4 \mu \mathrm{mol} / \mathrm{L})(5 \mu \mathrm{M})$ (Dojindo, Kumamoto, Japan) was loaded into the brain slice preparations for $90 \mathrm{~min}$. To monitor the $\left[\mathrm{Ca}^{2+}\right]_{\mathrm{i}}$, Fluo-3 fluorescence intensity (F525) was monitored using a photomultiplier under a confocal microscope (excitation wavelength $488 \mathrm{~nm}$; LSM5 PASCAL, Carl Zeiss, Germany). In our experiment, a lowmagnification ( $\times 2.5$ ) objective lens (FLUAR 2.5×, NA=0.12, Carl Zeiss, Germany) was used to perform Ca imaging of the entire hippocampal slice (Fig. 1). Fluo-3 fluorescent images $(512 \times 512$ pixels) were 
digitized and stored every $10 \mathrm{~s}$ for 50 min on a personal computer. Each F525 of CA1, CA2, CA3, DG, and EC regions was normalized (NF525) by the average value of F525 (from 0 to 5 min before AMPA or NMDA application) under offline analysis (Microsoft Excel, Microsoft Corporation, WA). During the Ca imaging, brain slices were continuously perfused $(2 \mathrm{~mL} / \mathrm{min})$ by extracellular solution containing $125 \mathrm{mM}$ $\mathrm{NaCl}, 2.5 \mathrm{mM} \mathrm{KCl}, 10 \mathrm{mM}$ D-glucose, $1.25 \mathrm{mM} \mathrm{NaH}_{2} \mathrm{PO}_{4}, 26 \mathrm{mM} \mathrm{NaHCO}_{3}, 2 \mathrm{mM} \mathrm{CaCl}_{2}, 1 \mathrm{mM} \mathrm{MgCl}_{2}$, and $1 \mu \mathrm{M}$ tetrodotoxin continuously bubbled by mixture gas $\left(95 \% \mathrm{O}_{2} ; 5 \% \mathrm{CO}_{2}\right)$ in the perfusion chamber (35 $\mathrm{mm} \mu$-dish, Ibidi GMBH, Gräfelfing, Germany). In the case of the NMDA application, concentration of $\mathrm{MgCl}_{2}$ was set to $0 \mathrm{mM}$ to prevent the Mg-dependent inhibition of NMDAR. Maximal value of NF525 was estimated as the maximal value of NF525 within 5 min after AMPA (Tocris, Bristol, UK) $(100 \mu \mathrm{M})$ or NMDA (Tocris, Bristol, UK) $(50 \mu \mathrm{M})$ application. We used the cyclothiazide (CTZ) $(100 \mu \mathrm{M})$ or glycine (Tocris, Bristol, UK) $(10 \mu \mathrm{M})$ to prevent the desensitization of AMPAR or to activate the NMDAR, respectively, and used the PER (Eisai Co., Ltd., Tokyo, Japan) (100 $\mu \mathrm{M})$, GYKI (Tocris, Bristol, UK) (100 $\mu \mathrm{M})$, or (2R)-amino-5-phosphonovaleric acid (APV) (Tocris, Bristol, UK) $(50 \mu \mathrm{M})$ as an antagonist of AMPAR or NMDAR respectively. We also used 1-naphthyl acetyl spermine (NASPM) (Tocris, Bristol, UK) $(20 \mu \mathrm{M})$ as an antagonist of $\mathrm{Ca}^{2+}$-permeable AMPAR [35] .

\section{Surface AMPAR subunit of cross-linking with $\mathrm{BS}^{3}$}

Hippocampal slices from 17-18-week-old male C57BL/6J background mice (CD, HFDs, and HFDs with PER treatment) were prepared as reported previously [34]. After preparing and recovering the hippocampal slice, we separated the CA1, CA2, CA3, DG, and EC regions from the hippocampal slice using an anatomical knife. CA1, CA2, CA3, DG, and EC regions were added to Eppendorf tubes (Eppendorf, Hamburg, Germany) containing ice-cold artificial cerebrospinal fluid (ARTCEREB; Otsuka Pharmaceutical Co., Tokyo, Japan) with $2 \mathrm{mM} \mathrm{BS}^{3}$ (Thermo Fisher Scientific, Wilmington, DE, USA). Incubation was performed for $30 \mathrm{~min}$ on ice. Cross-linking was terminated by quenching the reaction with $100 \mathrm{mM}$ glycine (10 $\mathrm{min}$ at $4^{\circ} \mathrm{C}$ ). Hippocampal subregions were resuspended in ice-cold lysis buffer containing protease and phosphatase inhibitors ( 25 mM HEPES, pH 7.4, $500 \mathrm{mM} \mathrm{NaCl}, 2 \mathrm{mM}$ EDTA, 1 mM DTT, $1 \mathrm{mM}$ phenylmethyl sulfonyl fluoride, $20 \mathrm{mM} \mathrm{NaF}, 1 \mathrm{mM}$ sodium orthovanadate, $10 \mathrm{mM}$ sodium pyrophosphate, $1 \times$ protease inhibitor mixture [Sigma-Aldrich, St Louis, MO], and 0.1\% Nonidet P-40 [v/v)]) and homogenized rapidly by sonicating for $5 \mathrm{~s}$. The total protein concentration of lysates was determined using the Lowry method. Samples were aliquoted ( $\sim 15$ aliquots per mouse) and stored at $-80^{\circ} \mathrm{C}$ for future analysis. BS ${ }^{3}$ samples were analyzed directly by SDS-PAGE without purification, and surface and intracellular bands were measured in the same lane, avoiding the need for normalization and increasing sample throughput. Total protein lysates $(40 \mu \mathrm{g})$ were loaded and electrophoresed on a $5-10 \% \mathrm{Tris}-\mathrm{HCl}$ gel (Thermo Fisher Scientific, Wilmington, DE) under reducing conditions, and proteins were transferred to nitrocellulose membranes (Thermo Fisher Scientific, Wilmington, DE) for immunoblotting. Membranes were blocked with a blocking reagent, Blocking One (Nacalai Tesque, Tokyo, Japan), for 30 min at room temperature. Membranes were then incubated with anti-GluA1 (1:1000; Merck Millipore, Burlington, MA) and actin (1:5000, Protein Teck, Chicago, IL, USA) overnight at $4^{\circ} \mathrm{C}$. Membranes were incubated for 30 min with HRP-conjugated anti-rabbit IgG (1:3,000; Cell Signaling Technology, Danvers, MA, USA) and 
washed extensively again in TBS-T. Membranes were immersed in chemiluminescence detecting substrate Chemi-Lumi One (Nacalai Tesque, Tokyo, Japan) for $1 \mathrm{~min}$, and then detected using a LuminoGraph1 imaging system (Atto, Tokyo, Japan). The surface and intracellular bands in each lane were analyzed using a CS Analyzer (Atto, Tokyo, Japan).

\section{RT-PCR}

Hippocampal slices of 17-18-week-old male C57BL/6J background mice (CD, HFDs, and HFDs with PER treatment) were prepared as reported previously [34]. After preparing and recovering the hippocampal slices, we separated the CA1, CA2, CA3, DG, and EC regions from the hippocampal slices using an anatomical knife from 57BL/6J background mice. The CA1, CA2, CA3, DG, and EC regions of mice were isolated and immediately lysed with $0.5 \mathrm{~mL}$ TRIzol RNA isolation reagent (Thermo Fisher Scientific, Wilmington, DE) in a disposable homogenize tube BioMasher2® (Nippi, Tokyo, Japan). Total RNA was extracted individually from each region according to the rest of the protocol for the TRIzol RNA isolation reagent provided by the manufacturer (Thermo Fisher Scientific, Wilmington, DE). Using the PrimeScript RT Reagent Kit (Takara, Shiga, Japan), $1 \mu \mathrm{g}$ of total RNA was reverse transcribed. An aliquot of the resultant cDNA was diluted 1:10 and added to a master mix of TB Green Premix ExTaq (Takara, Shiga, Japan), and real-time PCR was performed using a 7500 Fast Real-Time PCR system (Applied Biosystems, Foster City, CA, USA) according to the manufacturer's instructions. The real-time PCR conditions were as follows: $95^{\circ} \mathrm{C}$ for $30 \mathrm{~s}$, followed by 40 cycles of $95^{\circ} \mathrm{C}$ for $5 \mathrm{~s}$, and $60^{\circ} \mathrm{C}$ for $34 \mathrm{~s}$. The fluorescence intensity at every annealing step was captured, and threshold cycle time values were determined using the 7500 software. The relative beta-actin expression levels were further normalized to the respective control (vehicle group). The relative expression level of a target gene in each sample was determined using the standard curve and further normalized to that of $\beta$-actin in the same sample. The primer and probe sequences for these genes are shown in Table S5. We defined "GluA2's RNA amount/(GluA1+GluA3+GluA4's RNA amount) ratio" as $\mathrm{Ca}^{2+}$-permeability index.

\section{Sanger sequencing}

Templates for the Sanger sequencing of the GluA2 editing site were prepared by amplifying cDNA with the following primer pair: forward primer, GAGGAATTTGAAGATGGAAGAGA, and reverse primer, AGGAGGAGATGATGATGAGGGT. PCR products (10\%) were confirmed by agarose gel electrophoresis. After confirming the appropriate anticipated size, the PCR product was purified using the innuPREP PCRpure Lite Kit (Analytik Jena, Jena, Germany) according to the manufacturer's instructions before direct sequencing. Cycle sequencing was performed using the BigDye Terminator v3.1 Cycle Sequencing Kit (Applied Biosystems, CA, USA) and each of the primers mentioned above. Sanger sequencing analysis was performed using ABI3500 (Applied Biosystems, CA, USA) and sequence viewer software 4Peaks.

\section{Editing assays}

The Q/R editing state of GluA2 was analyzed using the QuantStudio ${ }^{\text {TM }}$ 3D digital PCR system with a TaqMan ${ }^{\circledR}$ custom SNP genotyping assay (Thermo Fisher Scientific, Wilmington, DE). TaqMan probes 
were synthesized by Thermo Fisher Scientific and VIC-labeled probe was used to detect non-edited (Q) sequences and FAM-labeled probe was used to detect edited (R) sequences ( 5 ' CATCCTTGCTGCATAAA 3' and 5' ATCCTTGCCGCATAAA 3', respectively). The following primer pairs for amplification were used for PCR reaction: mice forward primer 5' TTGGGATTTTTAATAGTCTCTGGTTTTCCTT 3' and mice reverse primer 5' GACCAACCTTGGCGAAATATCG 3'. cDNA of 5-50 ng was mixed with digital PCR master mix (Thermo Fisher Scientific, Wilmington, DE) and the mixed TaqMan probes were loaded onto the QuantStudio $^{\text {TM }}$ 3D digital PCR 20K Chip (Thermo Fisher Scientific, Wilmington, DE, USA) using an automatic chip loader according to the manufacturer's instructions. Loaded chips were amplified using the Gene Amp 9700 PCR system (Thermo Fisher Scientific, Wilmington, DE, USA) under the following conditions: $96^{\circ} \mathrm{C}$ for $10 \mathrm{~min}, 39$ cycles at $56^{\circ} \mathrm{C}$ for $2 \mathrm{~min}$, and $98^{\circ} \mathrm{C}$ for $30 \mathrm{~s}$, followed by a final extension step at $60^{\circ} \mathrm{C}$ for $2 \mathrm{~min}$. After amplification, the chips were imaged using a QuantStudio 3D Instrument (Thermo Fisher Scientific, Wilmington, DE, USA). For analysis of the chip data, QuantStudio 3D Analysis Suite Cloud Software (Thermo Fisher Scientific, Wilmington, DE) was used for the relative and quantitative data analysis.

\section{Golgi staining and acquisition of images of the whole hippocampus}

The coronal sectioned mice brains from Bregma $-0.94 \mathrm{~mm}$ to Bregma $-4.04 \mathrm{~mm}$ (including dorsal to ventral, medial to lateral whole hippocampus, $5 \mathrm{~mm}$ in width) was fixed by $4 \%$ paraformaldehyde for 2

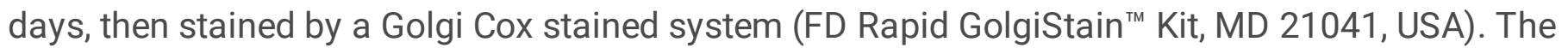
impregnated tissues were cut into $100 \mu \mathrm{m}$ sections and counterstained with crystal violet, and the total number of the $15 \mu \mathrm{m}$ spines in the apical dendrites of CA1 and DG, as well as the percentage of morphological spines (thin, stubby, and mushroom), were examined using Axio Observer Z1 (Carl Zeiss, Germany). Another set of paraformaldehyde-fixed $5 \mathrm{~mm}$ width coronal sections of the brain including the whole hippocampus was processed using the passive CLARITY technique method (PACT) [36] and examined through Lightsheet Z.1 (Carl Zeiss, Germany) using a 10X clearing objective, and 3D images of the whole hippocampus of Thy1-YFPH transgenic wild type mouse (The Jackson Laboratory, stock number: 003709, strain name: B6.Cg-Tg.(Thy1-YFP)16Jrs/J) [37, 38], HFD-fed mice, and HFD-fed mice with PER-treatment were obtained, respectively. We acquired the maximum intensity projection image along the z-, time-, or channel dimensions, which created an output image where the pixels contained the maximum value over all images in the stack at a particular pixel location. With the Z-stack function, a series of $X Y$ images in different focus positions can be acquired, resulting in a Z-stack. In this way, a 3D dataset $400 \mu \mathrm{m} \times 400 \mu \mathrm{m} \times 400 \mu \mathrm{m}$ from the specimen in each dorsal to lateral and medial to lateral CA1, CA3, and DG was obtained. The gallery view displays images from the z-stack within a time series.

\section{Quantitative analysis of Thy1-YFPH ${ }^{+}$-cell number}

$3 \mathrm{D}$ reconstruction was completed using the Arivis Vision four-dimensional (4D) software. Blob finder (filter) was used to the rounded 2D and 3D segments close to the sphere-like shapes in a noisy image. Using the Gaussian scale, we found the object seeds and a watershed algorithm to identify the object 
boundaries. We set the average size of the structure of interest to $20 \mu \mathrm{m}$ and the threshold to 5 . Highresolution rendering is an approach for visualizing the current view in a higher image and data resolution.

\section{Immunohistochemical analysis}

Mouse brains were perfusion-fixed in 4\% paraformaldehyde and embedded paraffin and cut into 4 micrometer-thick sections used for the immunohistochemical analysis of monoclonal antibodies for DCX (E-6 monoclonal, 1:50, Santa Cruz Biotechnology Inc., Dallas, USA), MAP2ab (AP-20 monoclonal, 1:100, SIGMA-ALDRICH, St. Louis, USA), GluA1 (AB1540 polyclonal, 1:100, EMD Millipore Corp., Burlington, USA), and GluA2 (AB1768-1 polyclonal, 1:5, EMD Millipore Corp., Burlington, USA), respectively.

\section{Quantitative immunostaining area by ZEISS ZEN Intellesis software.}

Acquisition of immunostained area in each MAP2, GluA1, and GluA2 expression area was performed using AxioVision (Carl Zeiss, Germany) microscopy (20X objective lens) with a ZVI format. Generating data image processing with image segmentation was performed using machine learning with ZEN Intellesis software. More than 1000 cells were examined in each group. One-way ANOVA was used to test the variance among the three groups, excluding data of 3SD or more. Then, we used the following sample numbers: HFD series: MAP2 CD $n=731$, HFDs $n=288$, HFD+PER $n=225$;GluA1 CD $n=220$, HFD $n=156$, HFD+PER n=94; GluA2 CD n=78, HFD n=114, HFD+PER n=182; GluA2/GluA1 CD n=78, HFD n=94, HFD+PER $n=94$. The Bonferroni method was used for post-hoc testing to analyze significant differences among the groups.

\section{RNA-seq analyses}

The sequenced raw RNA-seq fastq reads were aligned to the mouse GRCm38 (ensemble release 104, http://ftp.ensembl.org/pub/release-104/fasta/mus_musculus/dna/) genome using HISAT2 (v.2.2.0). The average mapping rate for all the samples was $94.21 \%$ (range $88.70-96.30 \%$ ). Aligned reads were quantified using Salmon (v.0.14.2), and TPM values were calculated using StringTie (v.2.1.2). Variant calling was performed according to GATK Best Practices for RNAseq short variant discovery using the GATK package (v.3.8). Adding read group information, sorting, marking duplicates, and indexing were carried out using Picard's tools. The GATK tool Split N Cigar Reads was used for splitting reads into exon segments and hard clipping any sequences overhanging into the intronic regions. Variant calling and filtration were performed using GATK HaplotypeCaller and VariantFiltration, respectively. Functional annotation of the output variant was performed using the SnpEff (v.4.3). The RNA-editing level was calculated as the ratio of the total number of reads aligned to the GluA2 R/Q editing site (chr3: 80706912) to the number of reads with T-to-C conversion at this site.

\section{Statistical analysis}

In the animal model experiments, one-way ANOVA followed by Bonferroni multiple comparisons test and/or two-tailed t-test were used for the statistical analysis. Statistical significance was set at $p<0.05$. In the human experiments, the relationship between BMI, GM volume of the whole brain, body weight, and percentage of correct responses to task conditions (new, lure, and same) was analyzed using partial 
correlation analysis. Differences between mean brain activity and correct answer rate under three (new, lure, and same) task conditions were analyzed using one-way ANOVA.

\section{Results}

\section{HFDs stimulate calcium entry through a-3-hydroxy-5-methyl-4-isoxazole propionic acid receptor (AMPAR) in mice hippocampus}

We first examined mouse obesity models treated with HFDs for 12 weeks after weaning (the composition of the diets [39] is shown in Table S1) and whether consumption of HFDs during the juvenile/adolescent periods altered the dynamics of calcium signaling in the hippocampus by direct measurement of intracellular calcium concentration $\left(\left[\mathrm{Ca}^{2+}\right]_{\mathrm{i}}\right)$ in acute brain slices in the hippocampus with perihippocampal regions (Fig. 1a, Movie S1). Mice fed with HFDs exhibited marked activation of calcium signaling through AMPAR by the application of $100 \mu \mathrm{M}$ AMPAR with $100 \mu \mathrm{M}$ cyclothiazide (CTZ, a channel desensitizer) in the entorhinal cortex (EC), cornu ammonis 1(CA1), CA2, CA3, and dentate gyrus (DG) estimated by maximal value of normalized F525 (NF525) (Fig. 1b, c, d and e, and Table S2).

\section{Inactivation of $\mathrm{N}$-methyl-D-aspartate (NMDA) receptors induced by intracellular $\mathrm{Ca}^{2+}$ through AMPAR}

In contrast, calcium signaling through $50 \mu \mathrm{M}$ NMDA with $10 \mu \mathrm{M}$ glycine (a channel coactivator) was inactivated in the hippocampus derived from the mice with HFDs, whereas control diet (CD)-fed mice exhibited activation through the hippocampus (Fig. 1f, g, h, i, and j; Table S2; and Movie S2). In the hippocampal slices derived from mice fed with HFDs plus perampanel (PER) (Fycompa ${ }^{\circledR}$, Eisai, Japan) ( $5 \mathrm{mg} / \mathrm{kg}$ per day, orally delivered by hydrogel), a novel noncompetitive AMPAR antagonist [16], we identified normalized glutamatergic transmission through AMPAR (Fig. 1d, Table S2), and simultaneous recovery of NMDAR-mediated signaling (Fig. 1i, Table S2, and Movie S3). A similar change in $\left[\mathrm{Ca}^{2+}\right]_{\mathrm{i}}$ through AMPAR was observed in HFD-fed mice for at least 7 days (Fig. S1a, b, c). Interestingly, these mice retained their activation through NMDAR. We found that a rise of $\left[\mathrm{Ca}^{2+}\right]_{i}$ through AMPAR was normalized by the treatment of PER (Fig. S1b ). These pathological AMPAR-mediated increases in $\left[\mathrm{Ca}^{2+}\right]_{i}$ were completely abolished by the addition of $100 \mu \mathrm{M}$ PER, and similarly, for other AMPAR antagonists such as

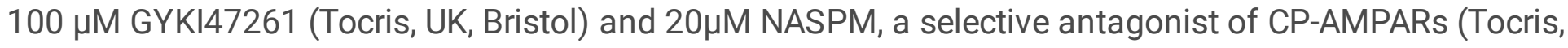
UK, Bristol) (Fig. S1d). As the signals through AMPAR and NMDAR of hippocampal slices derived from control mice fed with CDs plus PER did not show any significant difference compared with those fed with CDs alone (Fig. S9 a, b), PER treatment effects reversal of HFD-induced impairments as opposed to PER having effects on its own. We anticipated $\mathrm{Ca}^{2+}$-dependent inactivation $(\mathrm{CDI})[\mathbf{1 5 , 4 0 , 4 1 ]}$ observed in obesity mice is probably regulated by $\mathrm{Ca}^{2+}$-permeability of AMPARs and endogenous $\mathrm{Ca}^{2+}$ - buffer capacity. Indeed, AMPAR-mediated NMDAR inactivation was abolished in the presence of intracellular fast $\mathrm{Ca}^{2+}$ buffer BAPTA or in $\mathrm{Ca}^{2+}$-free extracellular solution (Fig. 2a, b). On the other hand, bath application of ionomycin $(1 \mu \mathrm{M})[42]$ with $2 \mathrm{mM} \mathrm{Ca}^{2+}$ induced CDI of NMDAR (Fig. 2c, d). These results suggest that HFD- 
induced impairments in NMDA receptor function in the hippocampus was mediated by increased $\mathrm{Ca}^{2+}$ permeability of AMPA receptors.

\section{Upregulation of calcium-permeable AMPAR by HFDs}

AMPAR is composed of four subunits, GluA1-4, which mediate fast excitatory neurotransmission and determine the functional properties of the AMPAR channel. $\mathrm{Ca}^{2+}$ permeability is regulated by the relative extent of GluA2 expression, which is essentially $\mathrm{Ca}^{2+}$ impermeability in relation to the extent of other $\mathrm{Ca}^{2+}$-permeable subunits of GluA1, GluA3, and GluA4, as well as the abundance of GluA2, and the status of GluA2 at the Q/R site within its re-entrant M2 membrane loop region edited by adenosine deaminase acting on RNA type 2 (ADAR2) responsible for adenosine (A) to inosine (I) editing of double-stranded RNA, and lastly the extent of GluA1 trafficking to the membrane $[7,8,14]$. NMDARs are heterometric composition assembled by NR1 and NR2A-D, and highly permeable to $\mathrm{Ca}^{2+}$. Properties of NMDAR are regulated by the relative extent of GluN2A-D subunits: open probability of GluN2A subunit containing NMDAR is 5 times or 50 times larger than that of the GluN2B or GluN2C/N2D subunit containing NMDAR respectively, single channel conductance of GluN2A/N2B subunit containing NMDAR is larger than that of the GluN2C/N2D subunit containing NMDAR respectively [43]. NMDARs play critical functions both in physiological and pathological processes in the CNS, and synaptic plasticity such as LTP, and LTD, which forms underlying mechanism for learning and memory associated with dendritic spine remodeling during their processes; LTP corresponds to mushroom and/or stubby spines, and LTD, shrinkage of their size. Their synaptic dysfunction induces cognitive decline in various CNS disorders including neurodegenerative, neuropsychiatric, demyelinating diseases, and so forth [44]. Thus, we sought to clarify the mRNA level of subunit composition of AMPAR, and NMDAR by real-time quantitative reverse transcription polymerase chain reaction (qRT-PCR) and editing level of GluA2 at the Q/R site in subregions of the hippocampus. We found significant elevation of GluA1, GluN1, and GluN2B expression in the CA3 region, and GluN2B expression in the DG region associated with HFD-fed mice $(p<0.05)$, respectively and a significant elevation of GluA2 in CA1 in HFD-fed mice with PER treatment $(p<0.05)$ (Fig. 3a). We introduced the index of calcium permeability of AMPA receptors (See Materials \& Methods). The reduction in the $\mathrm{Ca}^{2+}$-permeability index (Fig. 3b, Table S3) by HFD-fed mice $(n=5)$ was normalized to CD-fed mice level $(n=5)$ by treatment with PER $(n=5)$ (Fig. 3b, Table S3). These data would suggest that the extent of GluA1 expression of AMPAR in the hippocampus increased under the HFD condition compared with the CD condition and recovered under the HFD with PER condition. Upregulation of GluA1 content of AMPAR might play a crucial role in the upregulation of $\mathrm{Ca}^{2+}$ permeability of AMPARs in HFD mice (Fig. 1a, c). Cross-linking assays using BS ${ }^{3}$, a cross-linker that can be used to determine surface receptors and intracellular receptors, showed an elevation of three-fold the amount of GluA1 surface receptors in the HFD-fed group compared with the CD group $(n=4)$, and further declined to basal levels following treatment with PER $(n=4)$ in CA3 (Fig. 3c) (Fig. S4 a-c). In editing analysis at residue 607, we found that genomic glutamine ( $Q 607$ ) was converted to an arginine (R) codon of $>99.9 \%$ in both CD-fed and HFD-fed mice through CA1, CA2, CA3, DG, and EC (Fig. 3d) (Fig. S 4d), respectively. These findings altogether indicate the $\mathrm{Ca}^{2+}$-permeability change of AMPAR in HFD-fed mice was mainly 
dependent by the subunit composition change not dependent by the Q/R editing of GluA2 subunit. Up regulation of NMDA receptor mRNA in CA1,CA2, and CA3 but not DG and EC was detect in HFD-fed mice, although we could not find characteristic subunit compositional change. These results indicated that as reported by [15], increased $\mathrm{Ca}^{2+}$ - permeability of AMPA receptors may cause inactivity of NMDA receptors via the interaction of AMPA and NMDA receptors in HFD mouse. The change in the index of calcium permeability of AMPA receptors at the mRNA level are consistent with CP-AMPAR subunits composition and may cause secondary changes in NMDA receptor function.

\section{Structural reorganization of the brain of mice fed with HFDs}

We monitored the dietary intake and weight of mice after weaning ( 6 weeks) maintained on CD, HFDs, and HFDs with PER (Fycompa ${ }^{\circledR}$, Eisai, Japan) $(5 \mathrm{mg} / \mathrm{kg}$ per day) to 18 weeks, respectively (Fig. 4a, b). Although dietary caloric volume was not significantly different among the three groups, we found that the significant suppression of weight in the HFDs with PER group started from 16 weeks compared with the HFD alone group, although the significant body weight gain in the HFD group started from 8 weeks compared with the CD group. To clarify the effect of HFDs on cognitive behavior, we conducted an open field test (Fig. S2a-c), elevated plus maze test (Fig. S2 d, e), contextual fear conditioning test (Fig. S2f), novel object recognition test (Fig. 4c), and five-trial social interaction tests (social memory) (Fig. 4d). Among these tests, we found a significant difference (cognitive decline) between the CD and HFD groups in the latter two tests $(p<0.005)$. Hippocampal NMDARs were reported to play an important role in the retrieval of spontaneous object recognition memory $[3,4,45]$. Cognitive decline of object recognition memory in HFDs -fed mice should be due to the dysfunction of NMDA receptors in hippocampus (Fig. 1). As the hippocampal CA2 region has been reported to play an essential role in social memory [25] and social memory is dependent on NMDA receptor function [46], we found an abnormal social memory in HFD mice (Fig. 4d), which may be induced by the NMDAR dysfunction of the hippocampal CA2 region in HFD mice (Fig. 1). Indeed, PER treatment restored social memory disturbance in HFD-fed mice in accordance with the normalized NMDAR dependent $\left[\mathrm{Ca}^{2+}\right]_{i}$ signal of CA2 neuron. Water Maze test is strongly correlated with hippocampal synaptic plasticity and NMDA receptor function [47] and associative memory recall need to the activation of hippocampal CA3 NMDA receptors [48]. We found a reduced pattern completion ability (a retrieval ability to create explicit memory from partial cues of stored memory) in HFD mice, which was recovered in HFD with PER treatment mice associated with the recovery of $\mathrm{Ca}^{2+}$ entry through NMDAR of CA3 neuron (Fig. 1 \& Fig. 4e-g). Our data suggest that conversion from AMPAR to CP-AMPAR plays a key role in the disruption of pattern completion ability in HFD mice.

\section{Quantitative volumetric analysis of the brain}

Volumetric analysis is one of the fundamental approach to brain function [18]. In the quantitative volumetric analysis of the brain by 11.7 T magnetic resonance imaging (MRI) (Bruker BioSpec 117/11, Bruker BioSpin $\mathrm{GmbH}$, Germany), we found that all mice ( $n=6,16$ weeks old) fed with HFDs after weaning showed hydrocephalus (Fig. 5a), a notable sign of brain atrophy caused by the apparently reduction of total brain volume in every HFD-fed mice. Indeed, there was a significant reduction of GM and increase of 
WM in HFD mice $(n=6)$ compared to CD-fed mice $(p<0.01)$. Increase of WM, and disappearance of hydrocephalus in HFD mice was found by the PER treatment (Fig. 5a, and b). Furthermore, there was a significant reduction of the left CA1, bilateral CA3 and bilateral EC in HFD mice $(n=6)$ compared to CD-fed mice (Fig. 5b). We found that PER treatment in HFD-fed mice $(n=6)$ was significantly restored in the left and right sides of the hippocampal CA1 and DG and CA1, CA3, and DG regions, respectively (Fig. $5 \mathrm{~b}$ ). These volumetric increased hippocampal subregions were involved in the networks for associated and spatial memory, and we could see recue effect of these ability in mice treated with PER (Fig. 4d-e). In addition, Golgi- Thy1-eYFP transgenic mice [37] were adopted using clear lipid-exchanged acrylamidehybridized rigid imaging/immunostaining/in situ hybridization-compatible tissue-hydrogel (CLARITY) [49], and Z1 fluorescent microscopy (Carl Zeiss, Germany) was used to quantify neural cells in the hippocampal subregions. The reduced number of Thy- $-^{+}$-YFP neuronal cells in CA3, a center for associative memory recall, of HFD mice was significantly restored by PER treatment $(p<0.001)(n=4)$ (Fig. 5c, d, Movies S4-S 7) (Fig. S8): down regulation of neuronal number of hippocampal CA3 region may have a possible effect for the down regulation of NMDAR function in hippocampal CA3 region (Fig. 1) and the reduced pattern completion ability in HFD mice (Fig. $4 e, f$ and g). Our data indicated that hippocampal volume is not related to the cognitive function. In contrast, NMDARs was governed by the shape (length and radius) of the spine neck, spine neck morphology is related to the synaptic strength $[\mathbf{5 0}, \mathbf{5 1}]$. The spines on the dendrites are classified into mushroom type (with a large spine neck), thin type (with a medium size spine neck), and stubby type (without a spine neck) according to their shape. It has been reported that the density of AMPA receptors at synaptic sites depends on spine size $[\mathbf{5 2}, \mathbf{5 3}]$.

By Golgi-Cox impregnation staining, we found loss of integrity of dendritic arbors in CA1 in HFD mice, which was restored by PER (Fig. 6a, left upper panel). Similarly, the configuration of dendritic processes stained by anti-MAP2 antibody in CA1 showed the recovery of integrity of dendritic arbor by PER (Fig. 6a, left upper panel). There was no significant number of spines among CD, HFD and HFD plus PER treatment mice group, but a significant decrease of thin type spine, and a significant increase of stubby spine type was observed in HFD mice compared with CD mice. Furthermore, PER treatment in HFD mice increased mushroom spines, suggesting CP-AMPAR inhibits spine maturation. The immunoreactivity of GluA1 in CA1 was significantly enhanced in HFD mice but reduced with PER treatment, coordinating the elevation of GluA2/GluA1 ratio. In the DG, Golgi-stained cells were recovered in HFD mice treated with PER (Fig. 6a, left middle low), exhibiting simultaneously apical spine maturation (Fig. 6a, lower low). As shown in Fig. 6b, a significant increase in the immunoreactive area of GluA1 was observed in HFD mice, but not in GluA2. Since the ratio of GluA2 / GluA1 is significantly increased in HFD plus PER treatment mice compared with HFD mice, the change in the ratio of subunit composition contributes to the restoration of calcium permeability of AMPA receptor. In addition we also found that the increased doublecortin-positive cells in HFD plus PER hippocampus indicating regulation on calcium permeability by PER treatment induces neurogenesis in DG (Fig. 6c, left corner) and furthermore proceeds the maturation of $\mathrm{DCX}^{+}$-positive neural progenitors (Fig. S3a). These data suggest that synaptic immaturity, judged by increased stubby type, of the DG region under the HFD conditions was restored by the PER treatment (Fig. 6a). 


\section{Higher body mass index (BMI) impacts negatively on human gray matter (GM) volume}

To investigate the correlation between BMI and brain GM volume (gv), we enrolled 122 healthy Japanese subjects (including 84 [BMI: <25], 27 [BMI: 25-29.9], and 11 [BMI: >30] subjects) (Table S4) and applied volumetric analysis obtained by brain T2 MRI. We found a significant negative correlation between BMI and gv in men compared with women. Regarding higher BMI ( 30.2) and lower BMI ( 21.1) values in healthy individuals (Fig. 7a, b), we could observe a tendency for a decrease in gv with the BMI value. There was a significant negative correlation between "gv and ages," and "gv and body weights" (Fig. S5, S7 a-c). In contrast, we could not identify the significant correlation between "BMI and ages." However, there was a significant positive correlation between "BMI and body weights" (Fig. 7f) (Fig. S6). We also found a significant negative correlation between gv and BMI (Fig. $7 \mathrm{~g}$ ) using partial correlation analysis to minimize the effects of age and body weight.

Next, we examined the impact of BMl elevation on human hippocampal synaptic transmission by monitoring the fluctuation of blood oxygenation level-dependent (BOLD) response, hippocampal memory function of pattern completion, and pattern separation capabilities (the formation of distinct representations of similar inputs) using fMRI behavioral tasks (Fig. 7c-e) (Table S 6) $[17,18]$. Amplitude of Bold response was related to neural activity of both NMDAR and AMPAR in model animals [54], thus our data would indicate the modulation of AMPA and/or NMDAR would happen with the higher BMI condition in human. A negative correlation between $\mathrm{BMI}$ and pattern completion and ability for memory recall, whose basis of structural networks is CA3 and CA1 subregions that play a central role in ensemble dynamics [55], was observed in healthy human volunteers (Fig. 7h). Importantly, the overweight group did not reveal a significant cognitive decline compared with the control group (Fig. 7i); in contrast, people with $\mathrm{BMI}>30 \mathrm{~kg} / \mathrm{m}^{2}$ also showed a marked decline of pattern completion capabilities (Fig. 7j) (Table S7).

\section{Human pattern completion ability for memory recall is inversely correlated with higher BMI}

The significance of resting-state functional MRI (fMRI) for biomarkers of human cognition, especially in hippocampal memory function, is increasing [17]. Glutamatergic synapse could regulate cognitive function and brain connectivity [56], thus we clarified the functional network connectivity in resting-state in human. Among the intrinsically organized large-scale networks in resting-state fMRI data, default mode network dynamically controls the salience and central executive networks in healthy individuals, and this pattern of interaction has remained largely intact in overweight people; however, disorganization of this interaction in obese individuals results in cognitive decline (Fig. 8a). Lastly, we applied a quantitative analysis of human networks based on the graph theory in normal, overweight, and obese examinees. We created a functional connectivity map of each group by seed-based analysis and set the posterior cingulate cortex and precuneus in the default mode network and the anterior cingulate cortex and the cerebellar lobules Crus/in the hippocampal memory function as regions of interest [17]. The network connectivity varied among subjects in each classified group, as illustrated in Fig. 8b. Specific alternation 
of greater networks and between centrality mapping associated with the disruption of module autonomy became evident in the overweight and obese groups compared with the normal BMI group, resulting in failure of correct memory recall. The betweenness centrality of the anterior cingulate cortex and the paracingulate gyrus increased more than in the other two groups. Connectivity between different functional networks (DMN, SN, CEN, CBN, and VN) was extended in the obese groups. The total number of nodes in the normal weight, overweight, and obese groups was estimated to be 17,21 , and 23 , respectively (Fig. 8b, c).

\section{Discussion}

\section{A central role for CP-AMPAR in obesity-associated cognitive decline}

Here, we demonstrate that HFDs stimulate hippocampal calcium dynamics through AMPAR and subunit rearrangement from non-CP-AMPAR to CP-AMPAR, which leads to altered NMDAR calcium signaling to downregulate $\mathrm{Ca}^{2+}$ entry. Inactivated transmission through NMDAR function may correspond to cognitive decline. CP-AMPAR also triggered a reduction in hippocampal size and cell number, especially in CA3, dendritic integrity of $\mathrm{CA} 1$, and spine maturation in the DG region. Intake of HFDs for at least 7 days influenced the hippocampal memory circuit through calcium signaling. For this short-term feeding, calcium dynamics through NMDAR were maintained, but calcium entry through AMPAR became evident in the hippocampus. With intake for a long-term duration of more than 8 weeks after weaning with HFDs, calcium entry through NMDAR completely diminished in accordance with the alteration of calcium dynamics through AMPAR. Suppression of NMDAR-dependent calcium influx in synapses is modulated by intracellular calcium concentration irrespective of the source of calcium, such as AMPARs, voltagegated calcium channels, internal stores, and NMDAR [15]. Above all, AMPARs are colocalized with NMDAR and commit excitatory synaptic response on a tenths millisecond time scale, and HFDs induced an increase of calcium entry by these receptors, which finally resulted in reducing magnitude of calcium current through NMDARs reported to play a key role for the regulation of appetite and food preference [57] on to the induction of synaptic plasticity (LTP/LTD) [58] that stimulates a vicious cycle for binge eating, a reaction by failure in food restriction, and increased obesity [59] finally further accelerating cognitive decline.

\section{Alternation of glutamatergic synaptic transmission impacts on functional network connectivity in resting- state fMRI data}

Higher BMI is associated with cognitive disturbance in younger adults [60] in middle-aged adults, overweight and obesity increases the future risk of cognitive decline in old age. For people in old age, higher BMIs are paradoxically associated with better cognition and decreased mortality [61]. HFDs induce obesity, which leads to hypertension, type 2 diabetes, and cardiovascular events, and patients with these diseases suffer from an increased risk of cognitive decline $[62,63]$ which probably hinders effective treatment and disease control due to the failure of proper lifestyle management, including self-regulation of eating behavior. In medicine, developing an effective intervention for obesity-associated cognitive 
decline a key to better disease control. The correlation between the decreased resting-state activity in the precuneus and posterior cingulate and simultaneous anti-correlated inactivity of the dorsolateral prefrontal cortex and insula was disrupted in the brains of overweight and obese individuals (Fig. 8). A similar disruption was observed in subjects with metabolic syndrome [64].

\section{AMPAR is an attractive target for obesity-associated cognitive decline}

Of note, a high incidence of hydrocephalus was found among the early onset obese mice group. In humans, sustained obesity starts in early childhood between 2 and 6 years of age [65], and most children who are obese at that age are obese in adolescence. Proper and effective interventions are urgently needed for human health and disease. Importantly, application of HFDs with PER treatment restored the dysregulated calcium signaling through both AMPARs and NMDARs, while simultaneously inducing the recovery of brain size and behavioral memory capability. The prevention of weight gain in obesity induces health benefits. However, food restrictions that are reported to induce CP-AMPARs in the nucleus accumbens [66] usually rebound and result in weight gain and overeating. Taken together, these findings suggest that AMPARs are an attractive therapeutic target for human obesity-associated cognitive decline.

\section{Declarations}

\section{Data availability}

The datasets that support the findings of this study are available from the corresponding author upon reasonable request.

\section{Acknowledgments}

The authors wish to acknowledge Professor Kenzo Takahashi (University of the Ryukyus) for his critical review of our manuscript. The authors wish to acknowledge Professor Yamamoto, Chief Executive Officer of OIST's MRI system. Rodent MRI was performed at the University of the Ryukyus under the OIST Sign Cooperation Agreement. The authors wish to acknowledge Mr. Yasuhiko Sato and Mr. Masato Takiguchi (Carl Zeiss) for their special technical support and Mr. Keiki Oshiro for his secretarial assistance.

\section{Author Contributions}

YM contributed qRT-PCR, Q/R, rodent behavioral analysis, and planning of animal experimental design. KF contributed Immunohistochemical analysis. KH contributed Rodent MRI imaging. DU contributed NGS analysis. CK contributed Western blot analysis. MN contributed Human and rodent MRI imaging and network analysis. $\mathrm{HT}$ contributed $\mathrm{Ca}^{2+}$ imaging and editing of the manuscript. SH contributed $\mathrm{Z1}$ imaging, Golgi staining, project administration, funding acquisition, conceptualization and writing, and review and editing of the manuscript. All authors have no competing interests.

\section{Funding}


This work was supported by Grants-in-Aid for Scientific Research (B) (23390352) (to S.I.); Grants-in-Aid for Scientific Research (A) (17H01403) (to S.I.); Challenging Exploratory Research (24650168) (to S.I.) by the Ministry of Education, Culture, Sports, Science and Technology in Japan; the Industrial Disease Clinical Research Grants by the Ministry of Health, Labor and Welfare (14050101-01) (to S.I.); Special Account Budget for Education and Research (2011-2013, 2011-2015, 2014-2018, 2015-2019) (to S.I.); Grants for the Princess Takamatsu Cancer Fund (to S.I.); and the Grants for Takeda Science Foundation (to S.I.).

\section{Ethics Approval}

All animal experiments were performed in accordance with the guidelines of the Animal Experiment Ethics Committee of the University of the Ryukyus (approval number: A2019239).

All experiments were approved by the ethical committee of the University of the Ryukyus for medical and health research involving human subjects and were performed in accordance with guidelines of human experiment regulations at University of the Ryukyus (approval number:111).

\section{Consent to Participate}

Not applicable.

\section{Consent for Publication}

All authors have seen and approved the manuscript and contributed significantly to this work.

\section{Competing Interests}

The authors declare no competing interests.

\section{References}

1. Noble EE, Kanoski SE. Early life exposure to obesogenic diets and learning and memory dysfunction. Curr. Opin. Behav. Sci. 2016, 9, 7-14. doi:1016/j.cobeha.2015.11.014.

2. Hargrave SL, Jones S, Davidson TL. The outward spiral: A vicious cycle model of obesity and cognitive dysfunction. Curr. Opin. Behav. Sci. 2016, 9, 40-46. doi:1016/j.cobeha.2015.12.001.

3. Hwang L-L, Wang C-H, Li T-L, Chang S-D, Lin L-C, Chen C-P, Chen C-T, Liang K-C, Ho I-K, Yan W-S, Chiou L-C. Sex differences in high-fat diet-induced obesity, metabolic alterations and learning, and synaptic plasticity deficits in mice. Obesity (Silver Spring) 2010, 18, 463-469. doi: 1038/oby.2009.273.

4. Valladolid-Acebes I, Stucchi P, Cano V, Fernández-Alfonso MS, Merino B, Gil-Ortega M, Fole A, Morales L, Ruiz-Gayo M, Olmo ND. High-fat diets impair spatial learning in the radial-arm maze in mice. Neurobiol. Learn. Mem. 2011, 95, 80-85. doi: 1016/j.nlm.2010.11.007.

5. Fotuhi M, Do D, Jack C. Modifiable factors that alter the size of the hippocampus with ageing. Nat. Rev. Neurol. 8, 2012,189-202. doi: 1038/nrneurol.2012.27. 
6. Olmo ND, Ruiz-Gayo M. Influence of high-fat diets consumed during the juvenile period on hippocampal morphology and function. Front. Cell. Neurosci. 2018, 12, 439. doi: 3389/fncel.2018.00439.

7. Cull-Candy S, Kelly L, Farrant M. Regulation of $\mathrm{Ca}^{2+}$-permeable AMPA receptors: synaptic plasticity and beyond. Curr. Opin. Neurobiol. 2006, 16, 288-297.doi: 1016/j.conb.2006.05.012.

8. Liu SJ, Zukin RS. $\mathrm{Ca}^{2+}$-permeable AMPA receptors in synaptic plasticity and neuronal death. Trends Neurosci. 2007, 30, 126-134. doi: 1016/j.tins.2007.01.006.

9. Ishiuchi S, Tsuzuki K, Yoshida Y, Yamada N, Hagimura N, Okado H, Miwa A, Kurihara H, Nakazato Y, Tamura M, Sasaki T, Ozawa S. Blockage of $\mathrm{Ca}^{2+}$-permeable AMPA receptors suppresses migration and induces apoptosis in human glioblastoma cells. Nat. Med. 2002, 8, 971-978. doi: 1038/nm746.

10. Akamatsu M, Yamashita T, Hirose N, Teramoto S, Kwak S. The AMPA receptor antagonist perampanel robustly rescues amyotrophic lateral sclerosis (ALS) pathology in sporadic ALS model mice. Sci. Rep. 2016, 6, 28649. doi: 10.1038/srep28649.

11. Conrad KL, Tseng KY, Uejima JL, Reimers JM, Heng L-J, Shaham Y, Marinelli M, Wolf Formation of accumbens GluR2-lacking AMPA receptors mediates incubation of cocaine craving. Nature 2008, 454, 118-121. doi: 10.1038/nature06995

12. Khangura RK, Bali A, Jaggi AS, Singh N. Histone acetylation and histone deacetylation in neuropathic pain: an unresolved puzzle? Eur. J. Pharmacol. 2017, 795, 36-42. doi: 10.1016/j.ejphar.2016.12.001.

13. French JA, Krauss GL, Wechsler RT, Wang X-F, DiVentura B, Brandt C, Trinka E, O'Brien TJ, Laurenza A, Patten A, Bibbiani F. Perampanel for tonic-clonic seizures in idiopathic generalized epilepsy $A$ randomized trial. Neurology 2015, 85, 950-957. doi: 1212/WNL.0000000000001930.

14. Ozawa $\mathrm{Ca}^{2+}$-permeable AMPA receptors in central neurons. J. Physiol. 2009, 587, 1861-1862. doi: 10.1113/jphysiol.2009.169532.

15. Rozov A, Burnashev N. Fast interaction between AMPA and NMDA receptors by intracellular calcium. Cell Calcium 2016,60, 407-414. doi: 1016/j.ceca.2016.09.005.

16. Hibi S, Ueno K, Nagato S, Kawano K, Ito K, Norimine Y, Takenaka O, Hanada T, Yonaga M. Discovery of 2-(2-oxo-1-phenyl-5-pyridin-2-yl-1,2-dihydropyridin-3-yl)benzonitrile (perampanel): A novel, noncompetitive a-amino-3-hydroxy-5-methyl-4-isoxazolepropanoic acid (AMPA) receptor antagonist. J. Med. Chem. 2012, 55, 10584-10600. doi: 1021/jm301268u.

17. Shiroma A, Nishimura M, Nagamine H, Miyagi T, Hokama Y, Watanabe T, Murayama S, Tsutsui M, Tominaga D, Ishiuchi S. Cerebellar contribution to pattern separation of human hippocampal memory circuits. Cerebellum 2016, 15, 645-662.doi: 1007/s12311-015-0726-0.

18. Usugi R, Nishimura M, Ishiuchi S. Analysis of human hippocampal volumetry in relation to pattern separation ability in healthy young subjects. Brain Behav. 2020, 10, e01878. doi: 1002/brb3.1878.

19. Anai M, Funaki M, Ogihara T, Kanda A, Onishi Y, Sakoda H, Inukai K, Nawano M, Fukushima Y, Yazaki Y, Kikuchi M, Oka Y, Asano T. Enhanced insulin-stimulated activation of phosphatidylinositol 3kinase in the liver of high-fat-fed rats. Diabetes 1999,48, 158-169. doi: $\underline{2337 / \text { diabetes.48.1.158 }}$ 
20. Yonekawa T, Malicdan MCV, Cho A, Hayashi YK, Nonaka I, Mine T, Yamamoto T, Nishino I, Noguchi S. Sialyllactose ameliorates myopathic phenotypes in symptomatic GNE myopathy model mice. Brain 2014, 137, 2670-2679. doi: 1093/brain/awu210.

21. Ageta H, Murayama A, Migishima R, Kida S, Tsuchida K, Yokoyama M, Inokuchi K. Activin in the brain modulates anxiety-related behavior and adult neurogenesis. PLoS one 2008, 3, 1869. doi: 1371/journal.pone.0001869.

22. Huang T-N, Hsueh YP. Novel object recognition for studying memory in mice. Bioprotocol 2014, 4, Iss19. DOI: 10.21769/BioProtoc. 1249

23. Toda T, Noda Y, Ito G, Maeda M, Shimizu T. Presenilin-2 mutation causes early amyloid accumulation and memory impairment in a transgenic mouse model of Alzheimer's disease. J. Biomed. Biotechnol. 2011, doi: 10.1155/2011/617974.

24. Nakashiba T, Cushman JD, Pelkey KA, McBain CJ, Fanselow MS, Tonegawa S. Young dentate granule cells mediate pattern separation, whereas old granule cells facilitate pattern completion. Cell 149, 2012, 188-201. doi: 10.1016/j.cell.2012.01.046.

25. Hitti FL, Siegelbaum SA. The hippocampal CA2 region is essential for social memory. Nature 2014, 508, 88-92. doi: 1038/nature13028.

26. Chwang WB, O'Riordan K J, Levenson JM, Sweatt JD. ERK/MAPK regulates hippocampal histone phosphorylation following contextual fear conditioning. Learn. Mem. 2006, 13, 322-328. doi: 1101/Im.152906.

27. Dorr AE, Lerch JP, Spring S, Kabani N, Henkelman RM. High resolution three-dimensional brain atlas using an average magnetic resonance image of 40 adult C57BL/6J mice. Neuroimage 2008, 42, 6069. doi: 1016/j.neuroimage.2008.03.037.

28. Ullmann JFP, Watson C, Janke AL, Kurniawan ND, Reutens DC. A segmentation protocol and MRI atlas of the C57BL/6J mouse neocortex. Neuroimage 2013, 78, 196-203. doi: 1016/j.neuroimage.2013.04.008.

29. Richards TL, Grabowski TJ, Boord P, Yagle K, Askren M,. Mestre Z, Robinson P, Welker O, Gulliford D, Nagy W, Berninger V. Contrasting brain patterns of writing-related DTI parameters, fMRI connectivity, and DTI-fMRI connectivity correlations in children with and without dysgraphia or dyslexia. Neuroimage Clin. 2015, 8, 408-421. doi: 1016/j.nicl.2015.03.018. eCollection 2015.

30. Duvernoy HM, 2005, The human hippocampus: functional anatomy, vascularization and serial sections with MRI, Springer Verlag.

31. Whitfield-Gabrieli S, Nieto-Castanon A. Conn: a functional connectivity toolbox for correlated and anticorrelated brain networks. Brain Connect. 2012, 2, 125-141.doi: 1089/brain.2012.0073.

32. Rubinov M, Sporns 0 . Complex network measures of brain connectivity: Uses and interpretations. Neuroimage, 2010, 52, 1059-1069. doi: 1016/j.neuroimage.2009.10.003.

33. Xia M, Wang J, He Y. BrainNet Viewer: A network visualization tool for human brain connectomics. PLoS One 2013, 8, e68910.doi: 1371/journal.pone.0068910. Print 2013. 
34. Yao I, Takagi H, Ageta H, Kahyo T, Sato S, Hatanaka K, Fukuda Y, Chiba T, Morone N, Yuasa S, Inokuchi K, Ohtsuka T, Macgregor GR, Tanaka K, Setou M. SCRAPPER-dependent ubiquitination of active zone protein RIM1 regulates synaptic vesicle release. Cell 2007, 130, 943-957. doi: 1016/j.cell.2007.06.052.

35. Koike $\mathrm{M}$, lino $\mathrm{M}$, Ozawa S. Blocking effect of 1-naphthyl acetyl spermine on $\mathrm{Ca}^{2+}$-permeable AMPA receptors in cultured rat hippocampal neurons. Neurosci. Res. 1997, 29, 27-36. doi: 1016/s01680102(97)00067-9.

36. Treweek JB, Chan KY, Flytzanis NC, Yang B, Deverman BE, Greenbaum A, Lignell A, Xiao C, Cai L, Ladinsky MS, Bjorkman PJ, Fowlkes CC, Gradinaru V. Whole-body tissue stabilization and selective extractions via tissue-hydrogel hybrids for high-resolution intact circuit mapping and phenotyping. Nat. Protoc. 2015, 10, 1860-1896. doi: 10.1038/nprot.2015.122.

37. Feng G, Mellor RH, Bernstein M, Keller-Peck C, Nguye QT, Wallace M, Nerbonne JM, Lichtman JW, Sanes JR. Imaging neuronal subsets in transgenic mice expressing multiple spectral variants of GFP. Neuron 2000, 28, 41-51. doi: 1016/s0896-6273(00)00084-2.

38. Porrero C, Rubio-Garrido P, Avendaño C, Clascá F. Mapping of fluorescent protein-expressing neurons and axon pathways in adult and developing Thy1-eYFP-H transgenic mice. Brain Res. 2010, 1345, 59-72. doi: 10.1016/j.brainres.2010.05.061.

39. Anai M, Funaki M, Ogihara T, Kanda A, Onishi Y, Sakoda H, Inukai K, Nawano M, Fukushima Y, Yazaki Y, Kikuchi M, Oka Y, Asano T. Enhanced insulin-stimulated activation of phosphatidylinositol 3kinase in the liver of high-fat-fed rats. Diabetes 1999,48, 158-169. doi: 2337/diabetes.48.1.158

40. Legendre P, Rosenmund C, Westbrook GL. Inactivation of NMDA channels in cultured hippocampal neurons by intracellular calcium. J. Neurosci. 1993, 13, 674-684. doi: 10.1523/JNEUROSCI.13-0200674.

41. lacobucci GJ, Popescu GK. Ca ${ }^{2+}$-Dependent Inactivation of GluN2A and GluN2B NMDA Receptors Occurs by a Common Kinetic Mechanism. Biophys. J. 2020,118, 798-812. doi: 10.1016/j.bpj.2019.07.057.

42. Bickler PE, Fahlman CS. Moderate increases in intracellular calcium activate neuroprotective signals in hippocampal neurons. Neuroscience 2004, 127, 673-683. doi:

10.1016/j.neuroscience.2004.05.035.

43. Paoletti P, Bellone C, Zhou Q. NMDA receptor subunit diversity: impact on receptor properties, synaptic plasticity and disease. Nature Reviews Neuroscience 2013,14,383-400. doi: 10.1038/nrn3504.

44. Chidambaram SB, Rathipriya AG, Bolla SR, Bhat A, Ray B, Mahalakshmi AM, Manivasagam T, Thenmozhi AJ, Essa MM , Guillemin GJ, Chandra R, Sakharkar MK. Dendritic spines: Revisiting the physiological role. Prog. Neuropsychopharmacol. Biol. Psychiatry. 2019,92, 161-193. doi: 10.1016/j.pnpbp.2019.01.005.

45. Iwamura E, Yamada K, Ichitani Y. Involvement of hippocampal NMDA receptors in retrieval of spontaneous object recognition memory in rats. Behav. Brain Res. 2016, 307, 92-99. doi: 
1016/j.bbr.2016.03.048.

46. Yang J, Ma Q, Dincheva I, Giza J, Jing D, Marinic T, Milner TA, Rajadhyaksha A, Lee FS, Hempstead BL. SorCS2 is required for social memory and trafficking of the NMDA receptor. Mol Psychiatry. 2021, 6, 927-940. doi: 10.1038/s41380-020-0650-7.

47. Vorhees CV, Williams M. Morris water maze: procedures for assessing spatial and related forms of learning and memory. Nat. Protoc. 2006,1, 848-858. doi: 10.1038/nprot.2006.116.

48. Nakazawa K, Quirk MC, Chitwood RA, Watanabe M, Yeckel MF, Sun LD, Kato A, Carr CA, Johnston D, Wilson MA, Tonegawa S. Requirement for hippocampal CA3 NMDA receptors in associative memory recall. Science. 2002, 297,211-218. doi: 10.1126/science.1071795.

49. Chung K, Wallace J, Kim S-Y, Kalyanasundaram S, Andalman AS, Davidson TJ, Mirzabekov JJ, Zalocusky KA, Mattis J, Denisin AK, Pak S, Bernstein H, Ramakrishnan C, Grosenick L, Gradinaru V, Deisseroth K. Structural and molecular interrogation of intact biological systems. Nature 2013, 497, 332-337. doi: 1038/nature12107.

50. Araya R, Vogels TP, Yuste R. Activity-dependent dendritic spine neck changes are correlated with synaptic strength. Proc. Natl. Acad. Sci. U S A. 2014 Jul 15; 111(28): E2895-E2904. https://doi.org/10.1073/pnas.1321869111

51. Noguchi J, Matsuzaki M, Ellis-Davies GCR, Kasai H, Spine-Neck Geometry Determines NMDA Receptor-Dependent $\mathrm{Ca}^{2+}$ Signaling in Dendrites. Neuron. 2005, 46, 609-622. doi: 10.1016/j.neuron.2005.03.015.

52. Noguchi J, Nagaoka A, Watanabe S, Ellis-Davies GC, Kitamura K, Kano M, Kasai H. In vivo twophoton uncaging of glutamate revealing the structure-function relationships of dendritic spines in the neocortex of adult mice. Physiol. 2011, 589, 2447-2457. doi: 10.1113/jphysiol.2011.207100.

53. Matsuzaki M, Ellis-Davies GC, Nemoto T, Miyashita Y, lino M, Kasai H. Dendritic spine geometry is critical for AMPA receptor expression in hippocampal CA1 pyramidal neurons. Nature Neurosci. 2001, 4, 1086-1092. doi: 10.1038/nn736.

54. Gsell W, Burke M, Wiedermann D, Bonvento G, Silva AC, Dauphin F, Bührle C, Hoehn M, Schwindt W. Differential effects of NMDA and AMPA glutamate receptors on functional magnetic resonance imaging signals and evoked neuronal activity during forepaw stimulation of the rat. J. Neurosci. 2006 ,26, 8409-816. doi: 10.1523/JNEUROSCI.4615-05.2006.

55. Guzowski JF, Knierim JJ, Moser El. Ensemble dynamics of hippocampal regions CA3 and CA1. Neuron 2004, 44, 581-584. doi: 1016/j.neuron.2004.11.003.

56. Dauvermann MR, Lee G, Dawson N. Glutamatergic regulation of cognition and functional brain connectivity: insights from pharmacological, genetic and translational schizophrenia research. Br. J. Pharmacol. 2017, 174, 3136-3160. doi: 10.1111/bph.13919.

57. Sasaki T, Matsui S, Kitamura T. Control of appetite and food preference by NMDA receptor and its coagonist D-serine. Int. J. Mol. Sci. 2016,17, 1081. doi: 3390/ijms17071081.

58. Lüscher $\mathrm{C}$, Malenka RC. NMDA receptor-dependent long-term potentiation and long-term depression (LTP/LTD). Cold Spring Harb. Perspect. Biol. 2012, 4, a005710. doi: 1101/cshperspect.a005710. 
59. Kanoski SE, Davidson TL. Western diet consumption and cognitive impairment: Links to hippocampal dysfunction and obesity. Physiol. Behav. 2011, 103, 59-68.doi: 1016/j.physbeh.2010.12.003.

60. Hassing LB, Dahl AK, Pedersen NL, Johansson B. Overweight in midlife is related to lower cognitive function 30 years later: A prospective study with longitudinal assessments. Dement. Geriatr. Cogn. Disord. 2010, 29, 543-552. doi: 1159/000314874.

61. Jagust W, Harvey D, Mungas D, Haan M. Central obesity and the aging brain. Arch. Neurol. 2005, 62, 1545-1548. doi: 1001/archneur.62.10.1545.

62. Yates KF, Sweat V, Yau PL, Turchiano MM, Convit A. Impact of metabolic syndrome on cognition and brain: A selected review of the literature. Arterioscler. Thromb. Vasc. Biol. 2012, 32, 20602067.doi: 1161/ATVBAHA.112.252759.

63. Kopelman PG. Obesity as a medical problem. Nature 2000, 404, 635-643. doi: 1038/35007508.

64. Rashid B, Dev SI, Nicolette M, Schwarz F, Ferland T, Fortenbaugh FC, Milberg WP, McGlinchey RE, Salat DH, Leritz EC. Aberrant patterns of default-mode network functional connectivity associated with metabolic syndrome: A resting-state study. Brain Behav. 2019, 9, e01333. doi: 1002/brb3.1333

65. Geserick M, Vogel M, Gausche R, Lipek T, Spielau U, Keller E, Pfäffle R, Kiess W, Körner A. Acceleration of BMI in early childhood and risk of sustained obesity. N. Engl. J. Med. 2018, 379, 1303-1312. doi: 1056/NEJMoa1803527.

66. Ou-Yang DC, York PJ, Kleck CJ, Patel VV. Diagnosis and management of sacroiliac joint dysfunction. J. Bone Joint Surg. Am. 2017, 99, 2027-2036. doi: 2106/JBJS.17.00245.

\section{Figures}




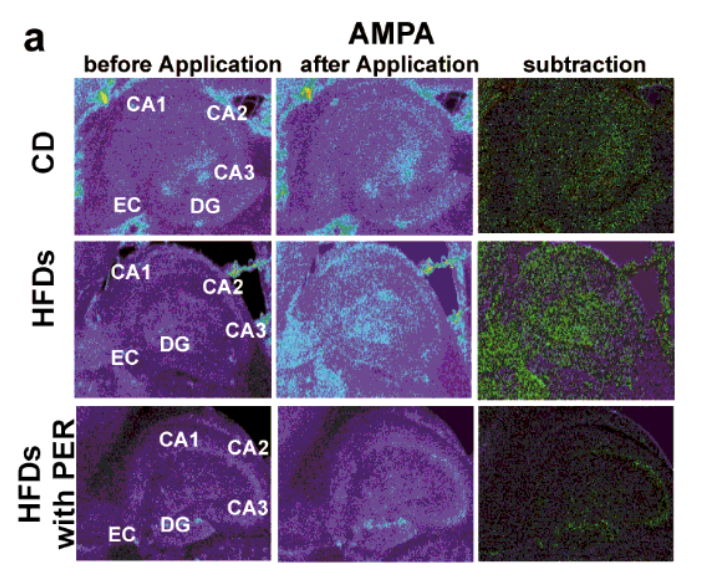

e
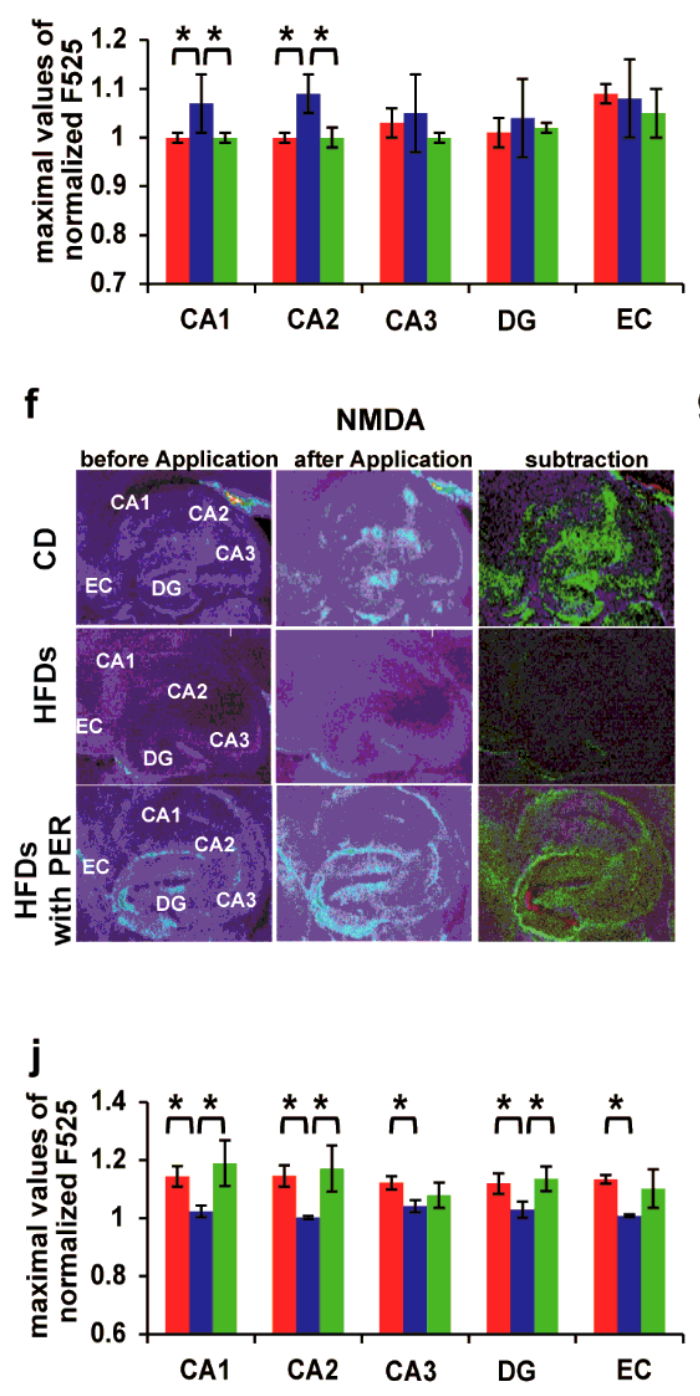

b $\quad C D$

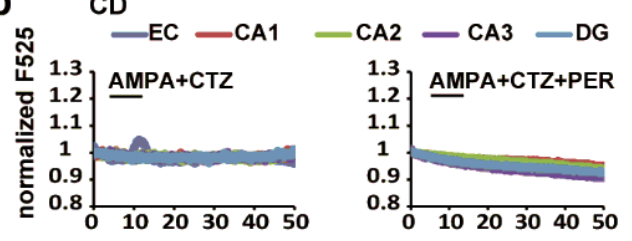

C HFDs

1.3 AMPA+CTZ

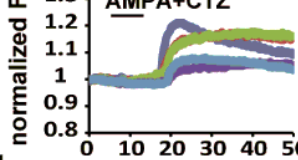

d
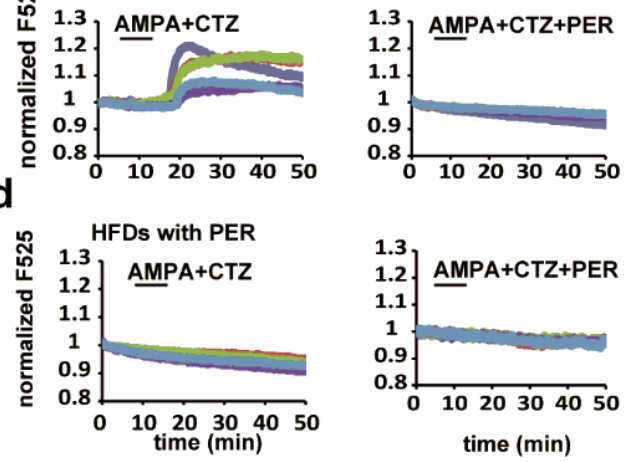

$\because \mathrm{CD}(\mathrm{N}=5)$

HFDs ( $\mathrm{N}=5)$

HFDs with PER ( $\mathrm{N}=5)$

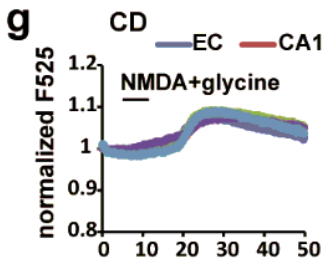

h
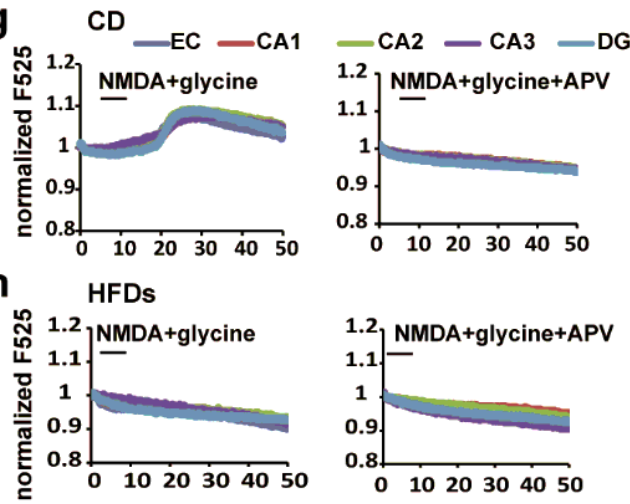

$i_{1.2}$ HFDs with PER
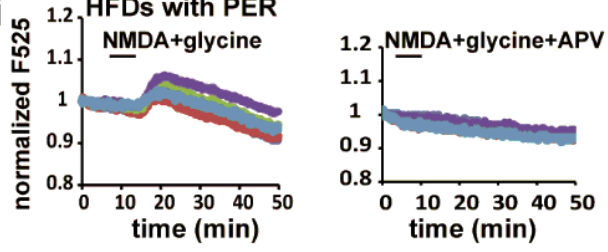

- $\mathrm{CD}(\mathrm{N}=5)$

- HFDs $(\mathrm{N}=5)$

HFDs with PER ( $\mathrm{N}=5)$

\section{Figure 1}

Perampanel (PER) rescued the alternation of AMPAR and NMDAR function in hippocampus of mice fed with HFDs.

a, Fluo-3 fluorescent image of hippocampus before and after AMPA with CTZ application under CD, HFD, and HFD with PER treatment conditions. Green subtraction images from after to before image. Mouse: 17 
weeks old; HFD or HFD with PER treatment: from 8 weeks to 17 weeks. b-d, AMPA dependent change of NF525 in hippocampal CA1, CA2, CA3, DG, and EC region; CD (b), HFD (c), and HFD with PER treatment (d) conditions, respectively. e, Averaged maximal value of NF525 after AMPA application from different slice preparations $(n=5) . *$ : $p<0.05$, two tailed t-test. $\mathbf{f}$, Fluo-3 fluorescent image of the hippocampus before and after NMDA with glycine application under CD, HFD, and HFD with PER treatment conditions and subtraction image. g-i, NMDA-dependent change of NF525 in hippocampal CA1, CA2, CA3, DG, and EC regions; CD (g), HFD (h), and HFD with PER treatment (i) conditions. j, Average maximal value of NF525 after NMDA application from different slice preparations $(n=5)$. **: $p<0.01 ; *$ : $p<0.05$, two tailed t-test. 

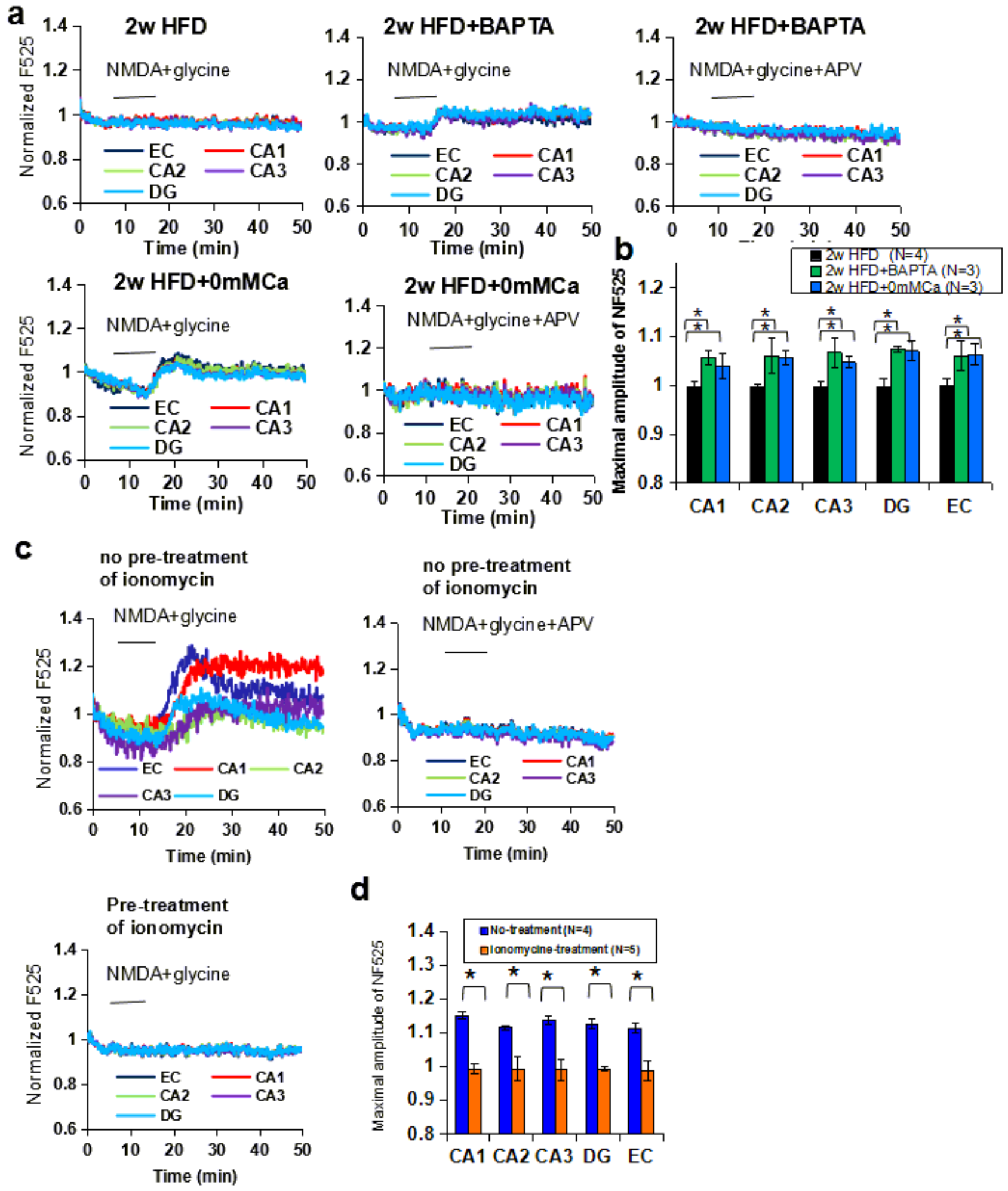

d

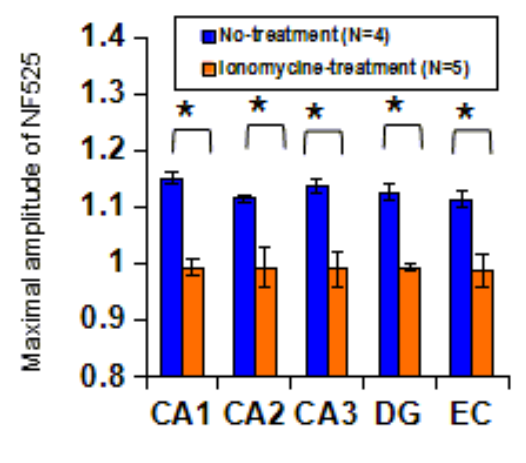

Figure 2

$\mathrm{Ca}^{2+}$-dependent inactivation of NMDAR under the HFD condition

a, Rescue effect by BAPTA-AM treatment or $\mathrm{Ca}^{2+}$ free conditions for HFD induced inactivation of NMDA receptor function. Hippocampal slice was pre-treated under the BAPTA-AM $3 \mu \mathrm{M}$ conditions or $\mathrm{Ca}^{2+}$ free conditions for $60 \mathrm{~min}$. before $\mathrm{Ca}^{2+}$ imaging experiments. 
b, Statistical comparison of NMDA receptor function among HFD $(n=4)$, BAPTA-AM treatment $(n=3)$ and

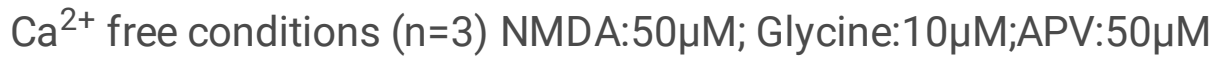

Mouse: 17 weeks old, from 15 week to 17 week HFD.

c, Pre-treatment (for $10 \mathrm{~min}$ ) of ionomycin $(1 \mu \mathrm{M})$ with $2 \mathrm{mM} \mathrm{Ca}^{2+}$ induced the inactivation of NMDAR function

d, Statistical comparison of NMDA receptor function between pre-treatment of ionomycin $(1 \mu \mathrm{M})$ with $2 \mathrm{mM} \mathrm{Ca}^{2+}$ group $(n=5)$ and no pre-treatment of ionomycin group $(n=4)$.

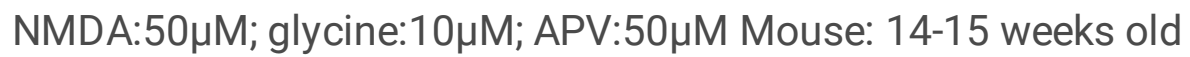

$* \llbracket p<0.05$ 


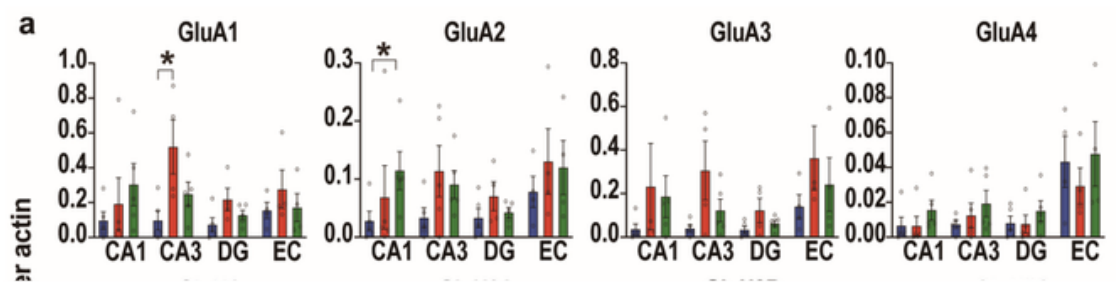

\section{Figure 3}

Transcriptional profiling in the hippocampal subregion, AMPAR and NMDAR subunit expression in mice fed with HFD.

a, Expressions of AMPAR subunits, NMDAR subunits, and ADAR2 in CA1, CA3, DG, and EC hippocampal subregions by qRT-PCR. Note the GluA1 at CA3 in mice fed with CD, HFDs, and HFDs with PER treatment. 
Mouse: 17 weeks old; HFD or HFD with PER treatment: from 8 weeks to 17 weeks. b, $\mathrm{Ca}^{2+}{ }^{2}$-permeability index of CA1, CA3, DG, and EC hippocampal subregions fed by CD, HFDs, and HFDs with PER treatment. c, Surface (center column) and intracellular (right column) expression of GluA1 protein in the hippocampal CA3 region in mice fed with HFDs increased, while that in mice fed with HFDs with PER treatment was downregulated. $\mathbf{d}$, Q/R editing analysis of CA1, CA2, CA3, DG, and EC hippocampal subregions in CD and HFD mouse. VIC: VIC-labeled probe to detect non-edited (Q) sequence, FAM: FAMlabeled probe to detect edited (R), Q \& R ratio (\%) (left upper and row) and copy number of each subregion (the box on the far right of the bottom row). Mouse: 17 weeks old; HFD or HFD with PER treatment: from 6 weeks to 17 weeks. 
a

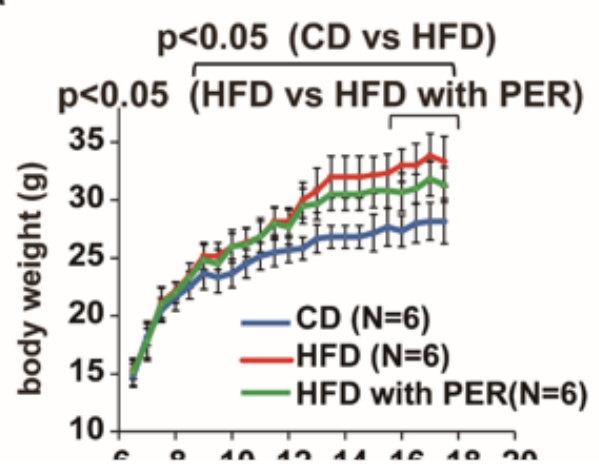

b

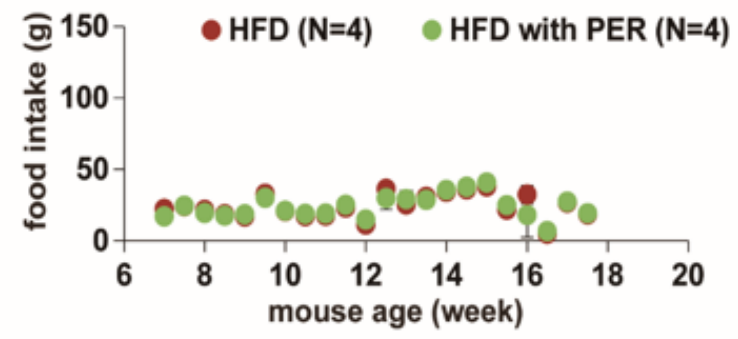

\section{Figure 4}

Behavioral changes in mice fed with HFD

a, Change in body weight of animals fed with CD, HFDs, and HFDs with PER treatment $(n=6)$. Mouse: 617 weeks old; HFD or HFD with PER treatment: from 6 weeks to 17 weeks. b, Food intake of animals fed with HFDs and HFDs with PER treatment $(n=4)$. 
c, Average investigation time of new object and familiar object in mice fed with $C D(n=11)$, HFDs $(n=12)$, and HFDs with PER treatment $(n=12)$. The symbol overlapping the bar graph indicates the individual data. Mouse: 12 weeks old; HFD or HFD with PER treatment: from 4 weeks to 12 weeks. $d$, Interaction time of five-trial social interaction tests on each day of testing in mice fed with CD, HFDs, and HFDs with PER treatment. Mouse: 16 weeks old; HFD or HFD with PER treatment: from 8 weeks to 16 weeks. Data are shown as mean \pm SD. $*$ : $p<0.05$; $* *$ : $p<0.01$, two tailed t-test. eg, Representative examples of the swim path to the phantom platform (blue) for the probe trials (end point of trajectory represents the start point) (e), probe trials with cue manipulation ( $\mathbf{f}$ ). Goal arrival time of the Morris water maze pattern completion test $(\mathbf{g})$ on each day of testing in mice fed with CD, HFD, and HFD + PER, respectively. Mouse: 17 weeks old. 


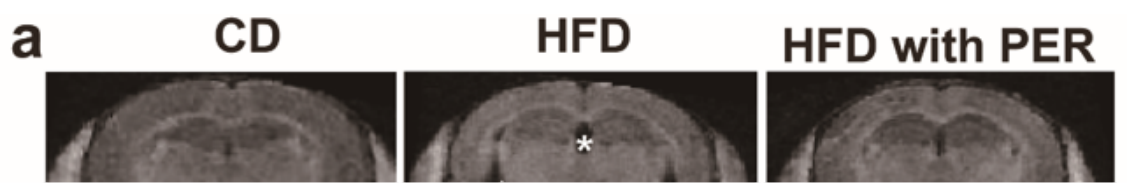

\section{Figure 5}

Brain weight and cell number alternation of the hippocampus by HFDs.

a, Coronal section of the MRI image under BL/6 (16 weeks old), HFDs, and HFDs with PER treatment conditions. White asterisk and diamond (HFD-fed mouse): dilated third and bilateral lateral ventricles. Right bar graph shows mean volume of total intracranial volume (TIV), whole brain gray matter (GM), and 
whole brain white matter (WM) per TIV of mouse. The Y-axis on the left and on the right show the volume of TIV $(\mathrm{mL})$ and the ratio of GM or WM to TIV, respectively. $\mathbf{b}$ Mean volume of GM per TIV of hippocampal CA1, CA3, DG, and EC. Left and right bar graph indicate left and right hippocampus, respectively. The Yaxis on the left shows the ratio of GM to TIV of CA1, CA3, and DG and on the right shows EC. c, d, 3D image of Thy-1 mouse (17 weeks old) hippocampus, length of cubic box; sagittal length axis (anterior to posterior): $4000 \mu \mathrm{m}$, width: $3000 \mu \mathrm{m}$, and height: $3000 \mu \mathrm{m}$ (c) and hippocampal CA1, CA3, and DG regions, each length of the cubic box is $400 \mu \mathrm{m}$ (width, depth, and height) (d) under BL/6, HFDs (from 4weeks to 17 weeks), and HFDs with PER (from 4weeks to 17 weeks) treatment conditions. Right bar graph showing cell density of the Thy-1+-FYP mouse hippocampal CA1, CA3, and DG regions, respectively.

\section{Figure 6}

\section{Micro level morphological alternation of the hippocampus by HFDs}

a-Cc, Golgi staining of CA1 and DG region (a, left column), schematic diagram of spine (a, right lower column)and percentage of thin, stubby, and mushroom-type spine (a, right upper column); anti-MAP2 Ab (antibody), anti-GluA1 Ab, and anti-GluA2 Ab staining (b, upper column); immuno-reactive area of antiMAP2 Ab (antibody), anti-GluA1 Ab, and anti-GluA2 Ab staining in hippocampal DG region and GluA2/GluA1 ratio (Bb, lower column); anti $\mathrm{DCX}^{+}$staining (c, left column); and number of $\mathrm{DCX}^{+}$positive cell/HFP (c, right column) in hippocampal DG region under BL/6, HFDs, and HFDs with PER treatment condition. Bar: $200 \mu \mathrm{m}$ (upper a), $100 \mu \mathrm{m}$ (middle a), $1 \mu \mathrm{m}$ (lower a), $50 \mu \mathrm{m}$ (b), and $100 \mu \mathrm{m}$ (left c) and $50 \mu \mathrm{m}$ (left c). Mouse age: 17 weeks old, HFD or HFD with PER treatment: from 4 weeks to 17 weeks. *, **, and $* \star *: p<0.05, p<0.01$, and $p<0.001$, respectively. 
a

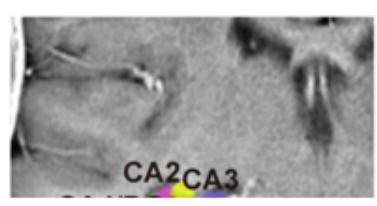

b

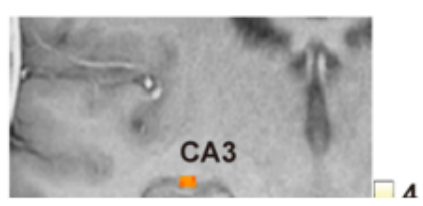

C

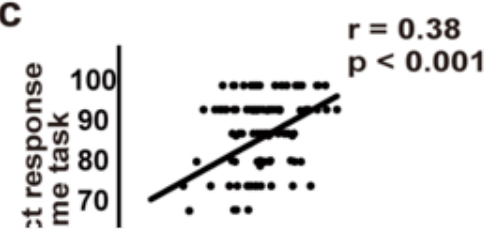

\section{Figure 7}

Increased BMI influences human memory function and synaptic fluctuation. a, Anatomy of a subregion of the right hippocampus on a T2-weighted image, indicated by cornu ammonis 1 (CA1), green; cornu ammonis 2 (CA2), magenta; cornu ammonis 3 (CA3), yellow; cornu ammonis 4 and dentate gyrus (CA4/DG), red; and subiculum (SUB), blue. $\mathbf{b}$, Correlation in the activation of CA3 and CA1 in the right hippocampus under recognition task (normal weight [n=84], overweight [n=27], and obese [n=11]). c-d, 
Correlation between percentage value of BOLD signal in CA3 of the right hippocampus and the percentage of correct response of the recognition task (c); percentage value of the BOLD signal of the bilateral hippocampal CA3 regions (d) (male: 52 ; female: 65 ; total: 117 ; average age $=37.8 \pm 19.6$ years, range 18-86 years). e, Mean value of the correct response rate under the recognition task. $\mathbf{f}, \mathbf{g}$, Correlation between $\mathrm{BMI}$ and the correct response rate under the recognition task $(r=-0.22, p<0.05)(f)$. Correlation between $\mathrm{BMI}$ and volume of whole brain gray matter $(r=-0.28, p<0.05)(\mathbf{g})$. In normal weight, overweight, and obese groups. h-j, Activation map and BOLD signal change in the left and right CA3, and the results of the correct response rate of the hippocampal memory task (New, Lure, Same) from left to right in normal weight (h), overweight (i, and obese (j) groups. Color bars: T-value. *: $p<0.001$.

\section{Figure 8}

Relationships between network connectivity and BMI in humans, a, DMN map (upper panel) and anticorrelation map to DMN (lower panel) on normal weight, overweight, and obesity groups. Color bars indicate T-value. b, Functional network connectivity among normal weight (left column), overweight (center column), and obese groups (right column). The diameter of each circle indicates the value of betweenness centrality. Blue, red, yellow, green, cyan, and orange nodes indicate Default mode network (DMN), Salience network (SN), Central executive network (CEN), Emotional network (EN), Cerebellar network (CBN), and Visual network (VN), respectively. c, Demonstrating the mean and standard deviation of the betweenness centrality and the degree of each node. a.u.: arbitrary unit.

\section{Supplementary Files}

This is a list of supplementary files associated with this preprint. Click to download.

- MovieS11.mp4

- MovieS12.mp4

- MovieS21.mp4

- MovieS22.mp4

- MovieS31.mp4

- Movies4.mp4

- Supplementallnformation.docx

- MovieS5.mp4

- Movies6.mp4

- Movies7.mp4

- Movies32.mp4 